\title{
A Transform on Classical Bounded Symmetric Domains Associated with a Holomorphic Discrete Series
}

Takeshi KAWAZOE

\author{
Keio University
}

\section{§ 1. Introduction.}

In order to explain the aim of this paper we shall look at an example by taking the Poincaré model of the hyperbolic plane $D$ and then consider its generalization.

1.1. Let $D$ be the open unit disk $|z|<1$ in $C$ with the usual manifold structure but given the Riemannian structure

$$
d s^{2}=\left(1-x^{2}-y^{2}\right)^{-2}\left(d x^{2}+d y^{2}\right) \quad(z=x+i y) .
$$

Let $G=S U(1,1)$ be the group of all $C$-linear transformations of $C^{2}$ preserving $\left|z_{1}\right|^{2}-\left|z_{2}\right|^{2}$ and of determinant one. Then each element $g$ of $G$ acts transitively on $D$ as an analytic automorphism of $D$ under

$$
z \rightarrow z \cdot g=(\bar{\alpha} z+\beta) /(\bar{\beta} z+\alpha)
$$

and $K=S O(2)$ is the subgroup of $G$ fixing 0 in $D$, so we have the identification: $D=S O(2) \backslash S U(1,1)$. If $f$ is a complex valued function on $D$, its Fourier transform $f^{\wedge}$ on $C \times \partial D, \partial D$ the boundary of $D$, is defined as follows:

$$
f^{\wedge}(\lambda, b)=\int_{D} f(z) e^{(i \lambda+1)\langle z, b\rangle} d z \quad(\lambda \in C, b \in \partial D)
$$

for which this integral exists. Here $\langle z, b\rangle$ is the number given by the relation

$$
e^{2\langle z, b\rangle}=\left(1-|z|^{2}\right) /|z-b|^{2} \quad(\lambda \in C, b \in \partial D) .
$$

Then the characterization of $L^{2}(D)^{\wedge}$, the set of Fourier transforms of $L^{2}$ functions on $D$, is well-known as the Plancherel theorem on $D$ (cf. [He], 
p. 33), though the one of $L^{p}(D)^{\wedge}(1 \leqq p<2)$ is an unsettled problem, even if the transform is the ordinary Fourier transform on a Euclidean space $\boldsymbol{R}$. Let $\left(V_{j, s}, L^{2}(\partial D)\right)(j=0,1 / 2$ and $s \in C)$ be the principal series representation of $G$ (cf. [Su], p. 212). Then by noting the identification $D \cong$ $K \backslash G$ we can rewrite (1.3) as

$$
f^{\wedge}(\lambda, b)=\int_{\theta} f(g) V_{0,(1 / 2)(-i \lambda+1)}\left(g^{-1}\right) 1(b) d g,
$$

where $f(g)$ is identified with $f(0 \cdot g), 1(b)$ is the constant function on $\partial D$ taking the value 1 and $d g$ is a $G$-invariant measure on $G$. In this sense (1.3) is based on the principal series of $G$, so we call (1.5) the Fourier transform associated with the principal series of $G$. Then the transform can be extended to all (non left $K$-invariant) functions on $G$ for which the integral exists.

Now we shall consider a transform which is defined by replacing the principal series in (1.5) with the discrete series of $G$. Let $\left(T_{n}, A_{2, n-1}(D)\right)$ $(n \in(1 / 2) Z$ and $n \geqq 1$ ) be the (holomorphic) discrete series representation of $G$ (cf. [Su], p. 237). Here $A_{p, r}(D) \quad(0<p<\infty$ and $r \in R)$ denotes the $\left(1-|z|^{2}\right)^{2 r}$-weighted $L^{p}$ Bergman space on $D$ (cf. [CR], §2). Then for a complex valued function $f$ on $G$ the Fourier transform $F_{n}(f)$ associated with the discrete series $T_{n}$ is defined by

$$
F_{n}(f)(z)=\int_{G} f(g) T_{n}\left(g^{-1}\right) 1(z) d g \quad(z \in D),
$$

where $1(z)$ is the constant function on $D$ taking the value 1 . Unlike the previous case, for a fixed $n$, we can obtain a characterization of $F_{n}\left(L^{p}(G)\right)(1 \leqq p \leqq 2)$, the set of $F_{n}$-Fourier transforms of $L^{p}$ functions on $G$, as follows:

$$
\text { If }(n, p) \neq(1,1) \text {, then } F_{n}\left(L^{p}(G)\right)=A_{p,(1 / 2) n p-1}(D)
$$

and

$$
\text { If }(n, p)=(1,1) \text {, then } F_{1}\left(L^{1}(G)\right)=H_{1,0}(D) \text {, }
$$

where $H_{1,0}(D)$ is the space of all holomorphic functions $F$ on $D$ such that $\partial F / \partial z$ belongs to $A_{1,0}(D)$ (see [Ka] and Theorem 5.5). This fact is a great difference between the transforms (1.5) and (1.6), and essentially this difference is due to the existence of a reproducing property of holomorphic functions on $D$.

The Plancherel theorem for $G=S U(1,1)$ (cf. [Su], p. 344) implies that each function $f$ in $L^{p}(G) \cap L^{2}(G)(1 \leqq p \leqq 2)$ has a decomposition like 
$f=f_{p}+{ }^{\circ} f$, where $f_{p}$ consists of wave packets and ${ }^{\circ} f$ is a linear combination of matrix coefficients of the discrete series of $G$. Then it easily follows from the definitions of (1.5) and (1.6) that for $f \in L^{p}(G) \cap L^{2}(G)$

$$
f^{\wedge}(\lambda, b)=f_{p}^{\wedge}(\lambda, b) \text { and } F_{n}(f)(z)=F_{n}\left({ }^{\circ} f\right)(z) .
$$

Moreover, $F_{n}$ has its support on a linear combination of the matrix coefficients of $T_{n}$ whose left $K$-type is just the lowest $K$-type of $T_{n}$, that is, if $\psi_{n}$ denotes the normalized matrix coefficient of $T_{n}$ corresponding to the lowest $K$-type, then it follows that

$$
F_{n}\left({ }^{\circ} f\right)=F_{n}\left(\psi_{n} *^{\circ} f\right)=F_{n}\left(\psi_{n} * f\right) \quad\left(f \in L^{p}(G) \cap L^{2}(G)\right) .
$$

Since $\psi_{n} * f$ belongs to $L^{2}(G)$ for $f \in L^{p}(G)$ (see [KS], Theorem 9, [Co] and Proposition 3.10), the right hand side of (1.9) is well-defined for $f \in L^{p}(G)$ and especially, $\psi_{n} * f$ is a linear combination of matrix coefficients of $T_{n}$. Then by approximating $L^{p}(G)$ with $L^{p}(G) \cap L^{2}(G)$ we see that

$$
F_{n}(f)=F_{n}\left(\psi_{n} * f\right) \quad\left(f \in L^{p}(G)\right)
$$

(see $[\mathrm{Ka}]$ and $\S 4$ ). Therefore, we can say that in (1.7) the transform $F_{n}$ characterizes a "discrete part" of $L^{p}(G)$, the set of all $L^{2}$ functions on $G$ being of the form $\psi_{n} * f$ for $f \in L^{p}(G)$, as a space of holomorphic functions on $D$. The fact that the discrete part $\psi_{n} * f$ of $f \in L^{p}(G)$ is in $L^{2}(G)$ implies that $F_{n}(f)$ has a reproducing property on $D$, and if the discrete part is in $L^{p}(G)$, the characterization of $F_{n}\left(L^{p}(G)\right)$ is given by the first case of (1.7). On the other hand the discrete part is not always in $L^{p}(G)$. This exceptional case, $n=p=1$, corresponds to the second characterization in (1.7), which is related with classical analysis of fractional derivatives of holomorphic functions on $D$ (see [Ka] and $\S 6$ ).

1.2. We shall explain briefly the contents of this paper. Let $G$ be a connected semisimple Lie group with finite center and $K$ a maximal compact subgroup of $G$. Then the Fourier transform associated with the principal series has been investigated by various people; for example the Plancherel theorem for $I^{2}(G), K$-biinvariant $L^{2}$ functions on $G$, is obtained by Harish-Chandra (cf. [Wa], p. 353). On the other hand, the Fourier transform (1.6) associated with the discrete series is not paid any attention, although the operator valued transform, which is defined by dropping $1(z)$ in (1.6), was investigated as a function on $n$ in [KS], $\S 9$. So, our aim is to generalize the above result (1.7) on $D$ to other symmetric spaces $G / K$ when $G$ has the discrete series. However, because of difficulty in calculating an explicit number (see Lemma 4.4) we assume 
that $\Omega^{\sim}=G / K$ is one of the classical bounded symmetric domains listed in Table 1 (cf. $[\mathrm{Hu}]$ and $[\mathrm{Kn}])$, and that the discrete series $T$, which will

TABLE 1

\begin{tabular}{clll}
\hline Type & \multicolumn{1}{c}{$\boldsymbol{G}$} & \multicolumn{1}{c}{$\boldsymbol{S}$} & \multicolumn{1}{c}{$\Omega=\log \left(\Omega^{\sim}\right)$} \\
\hline I & $S U(m, n)$ & $S(U(m) \times U(n))$ & $\left\{z \in M_{m m}(C) ; I_{m}-z \bar{z}^{\prime}>0\right\}$ \\
II & $S p(n, R)$ & $U(n)$ & $\left\{z \in M_{n n}(C) ; I_{n}-z \bar{z}>0, z=z^{\prime}\right\}$ \\
III & $S O^{*}(2 n)$ & $S p(n) \cap S O(2 n)$ & $\left\{z \in M_{n n}(C) ; I_{n}+z \bar{z}>0, z=-z^{\prime}\right\}$ \\
IV & $S O(n, 2)$ & $S O(n) \times S O(2)$ & $\left\{z \in C^{n} ;\left|z z^{\prime}\right|^{2}+1-2 z \bar{z}^{\prime}>0,\left|z z^{\prime}\right|<1\right\}$ \\
\hline
\end{tabular}

be used to define the desired transform $F_{T}$, is the holomorphic one whose lowest $K$-type $\tau$ is one dimensional representation of $K$ (see [Kn] and Table 3 in $\S 3$ ). Then under these assumptions we shall prove that $F_{T}$ characterizes exactly a discrete part of $L^{p}(G)(1 \leqq p \leqq 2)$ as a space of holomorphic functions on $\Omega=\log \left(\Omega^{\sim}\right)$.

The Fourier transform $F_{T}$ associated with $T$ is defined in the same way we do on $D$ (see (1.6) and (4.2)). Then as in the case of $\Omega=D$, to characterize the image of $L^{p}(G)$ we must prepare two types of function spaces on $\Omega$ consisting of holomorphic functions on $\Omega$. The selection of type is decided according to the $L^{p}$ integrability of the matrix coefficients of $T$; so let $\psi$ be the matrix coefficient corresponding to the lowest $K$ type $\tau$, and we say that $(\psi, p)$ is regular if $(\psi, p)$ satisfies the following condition:

$$
\psi \in L^{p}(G) .
$$

Then if $(\psi, p)$ is regular, the image $F_{T}\left(L^{p}(G)\right)$ coincides with an $L^{p}$ weighted Bergman space on $\Omega$, and if it is not regular, it coincides with a space defined by using a generalized fractional derivatives of holomorphic functions on $\Omega$ (see (5.1), (5.2) and Theorem 5.5).

In $\S 2$ we shall recall some basic properties of bounded symmetric domains $\Omega$ and holomorphic discrete series $T$ of $G$ (cf. [Kn]), and in $\S 3$ we shall obtain an $L^{p}$ estimate of the convolution of a matrix coefficient of $T$ and $L^{p}$ functions on $G$ (see Proposition 3.10). Essentially, this estimate is due to the two lemmas obtained in [CR] for the Bergman kernel on $\Omega$. Translating this $L^{p}$ boundedness, we can obtain the characterization in the case of regular (see Proposition 3.9 and Theorem $5.5(1))$.

When $(\psi, p)$ is not regular, the above $L^{p}$ estimate does not hold and the matrix coefficient of $T$ does not belong to $L^{1}(G)$. However, we see that the matrix coefficient is a "discrete part" of an $L^{1}$ function on $G$ (see Lemma 4.2). By using this fact we shall define the fractional 
derivatives of holomorphic functions on $\Omega$ (see Lemma 4.4 and (5.1)), and then define a space consisting of holomorphic functions on $\Omega$ (see (5.2)). This space is the desired one that characterizes the Fourier transform $F_{T}\left(L^{p}(G)\right)$ in the case of non regular (see Theorem $5.5(2)$ ).

In the last $\S 6$ we shall consider the continuation of the discrete parameter and give an application of the exact characterization of $F_{T}\left(L^{p}(G)\right)$. Actually, when a holomorphic function $u$ on $\Omega$ belongs to a weighted Bergman space, we can decide the weighted Bergman space to which the generalized fractional derivative of $u$ belongs (see Theorem 6.1). In the case of $\Omega=D$ this result was obtained in [DRS], Theorem 5.

\section{§ 2. Notation.}

We shall recall some basic facts about the bounded symmetric domains and holomorphic discrete series representations, which are summarized in $[\mathrm{Kn}]$.

2.1. Let $G$ be a simple matrix group and $K$ a maximal compact subgroup of $G$, and let us suppose that $K \backslash G$ is Hermitian. Let $g$ and $t$ denote the corresponding Lie algebras, and $\mathfrak{h}$ a maximal abelian subalgebra of $\mathfrak{t}$. Then $\mathfrak{h}$ is also a maximal abelian subalgebra of $\mathfrak{g}$. For an algebra $a$ of matrices the complexification is denoted by $\mathfrak{a}_{c}$. Let $\Sigma=\Sigma\left(\mathfrak{g}_{c}, \mathfrak{h}_{c}\right)$ be the set of non zero roots $\alpha$ for the pair $\left(\mathfrak{g}_{c}, \mathfrak{h}_{c}\right)$ and let $\mathfrak{g}_{\alpha}$ be the corresponding root space. We choose an ordering in which every non compact positive root is larger than every compact root. Let $\Sigma^{+}$and $\Sigma_{n}$ denote the sets of positive roots and non compact roots in $\Sigma$ respectively, and let $\Sigma^{-}=\Sigma-\Sigma^{+}$and $\Sigma_{k}=\Sigma-\Sigma_{n}$. Then we let

$$
\mathfrak{n}_{c}^{-}=\sum_{\alpha \in \Sigma^{-}} \mathfrak{g}_{\alpha}, \quad \mathfrak{b}=\mathfrak{h}_{c}+\mathfrak{n}_{c}^{-}, \quad \mathfrak{p}^{+}=\sum_{\alpha \in \Sigma^{+} \cap \Sigma_{n}} \mathfrak{g}_{\alpha} \quad \text { and } \quad \mathfrak{p}^{-}=\sum_{\alpha \in \Sigma^{-} n \Sigma_{n}} \mathfrak{g}_{\alpha} .
$$

Let $G_{c}$ be an analytic subgroup of matrices with the Lie algebra $g_{c}$, and $N_{c}^{-}, B, P^{+}, P^{-}, K_{c}, T_{c}$ the subgroups of $G_{c}$ corresponding to $\mathfrak{n}_{c}^{-}, \mathfrak{b}, \mathfrak{p}^{+}, \mathfrak{p}^{-}$, $\mathfrak{t}_{c}, \mathfrak{h}_{c}$ respectively. It is known that the multiplication $P^{-} \times K_{c} \times P^{+} \rightarrow G_{c}$ is one to one, holomorphic and regular, that $B G$ is open in $G_{c}$ and there exists a bounded set $\Omega^{\sim}$ in $P^{+}$such that

$$
B G=P^{-} K_{c} G=P^{-} K_{c} \Omega^{\sim} \subset P^{-} K_{c} P^{+} .
$$

Let $p^{+}(x)$ refer to the $P^{+}$-component of $x$ in $P^{-} K_{0} P^{+}$. Then $G$ acts transitively on $\Omega^{\sim}$ by $z \cdot g=p^{+}(z g)$ as a holomorphic automorphism on $\Omega^{\sim}$, and $G \cap P^{-} K_{c}=K$ is the subgroup of $G$ fixing 1 in $\Omega^{\sim}$, so we have the identification 


$$
\Omega^{\sim}=K \backslash G
$$

Let $\log$ be the inverse mapping of the exponential map exp: $\mathfrak{p}^{+} \rightarrow P^{+}$and let $\Omega=\log \left(\Omega^{\sim}\right)$.

2.2. Let $\Lambda \in\left(\mathfrak{h}_{c}\right)^{*}$, the set of complex linear functionals on $\mathfrak{h}_{c}$, and suppose that $\Lambda$ is an integral form on $\mathfrak{h}_{c}$ dominant with respect to $\mathfrak{k}$. Let $\left(\tau_{A}, V_{A}\right)$ be an irreducible unitary representation of $K$ (and $K_{0}$ by holomorphical extension) with highest weight $\Lambda$, and let $\phi_{\Lambda}$ be a normalized highest weight vector and $\chi_{A}$ the associated character of $\tau_{A}$. Then we define

$$
\psi_{A}(x)=\left(\tau_{A}(\mu(x)) \phi_{A}, \phi_{A}\right) \quad\left(x \in P^{-} K_{c} P^{+}\right),
$$

where $\mu(x)$ refers to the $K_{c}$-component of $x$ in $P^{-} K_{c} P^{+}$, and for a complex valued function $f$ on $G_{c}$, we define a projection operator which maps $f$ to the left $\tau_{A}$-component of $f$ as

$$
E_{A}(f)(x)=\operatorname{dim} V_{A} \cdot \int_{K} \chi_{\Lambda}\left(k^{-1}\right) f(k x) d k \quad\left(x \in G_{c}\right)
$$

Since $\Lambda$ is integral,

$$
\xi_{\Lambda}(h)=\exp \Lambda(\log (h)) \quad\left(h \in T_{c}\right)
$$

is a well-defined character of $T_{c}$ and by setting $\xi_{A}(n)=1$ for $n$ in $N_{c}^{-}$, we can extend it to a holomorphic character of $B=N_{c}^{-} T_{c}$. We define

$$
\begin{aligned}
\Gamma(\Lambda)=\{f: B G \rightarrow C ; & \text { (1) } f \text { is holomorphic on } B G, \\
& \text { (2) } \left.f(b x)=\xi_{A}(b) f(x) \text { for } b \in B, x \in B G\right\}
\end{aligned}
$$

and for $0<p<\infty$

$$
\begin{aligned}
& A_{\Lambda}^{p}=\left\{f \in \Gamma(\Lambda) ;\|f\|_{p}^{p}=\int_{G}|f(g)|^{p} d g<\infty\right\}, \\
& A_{\Lambda}^{p}(G)=\left\{f \mid G ; f \in A_{\Lambda}^{p}\right\} .
\end{aligned}
$$

Some known properties of $\psi_{A}$ are the following

LEMMA 2.1 (cf. [Kn]). (1) $E_{\Lambda} \psi_{\Lambda}=\psi_{A}$ as a function on $P^{-} K_{c} P^{+}$.

(2) The restriction to $B G$ of $\psi_{A}$ belongs to $\Gamma(\Lambda)$.

(3) $\psi_{A}\left(x^{-1}\right)=\bar{\psi}_{1}(x)(x \in G)$.

(4) If $\langle\Lambda+\rho, \alpha\rangle<0$ for every non compact positive root $\alpha$, then $\left\|\psi_{A}\right\|_{2}<\infty$, in particular, $\psi_{A}$ belongs to $A_{\Lambda}^{2}$. 
REMARK 2.2. The constant $c_{A}=\left\|\psi_{A}\right\|_{2}$ in Lemma 2.1 (4) was explicitly calculated by $[\mathrm{HC}]$, p. 608 .

2.3. Let $w(z)$ be a positive function on $\Omega$. We define the $w$-weighted $L^{p}(0<p<\infty)$ Bergman space on $\Omega$ as follows:

$$
\begin{aligned}
A_{w}^{p}(\Omega)=\{F: \Omega \rightarrow C ; & \text { (1) } F \text { is holomorphic on } \Omega, \\
& \left.(2)\|F\|_{p, w}^{p}=\int_{\Omega}|F(z)|^{p} w(z) d z<\infty\right\},
\end{aligned}
$$

where $d z$ is a Euclidean measure on $\Omega$. Let $B(z, \bar{w})$ denote the Bergman kernel on $\Omega$ listed below (cf. [CR] and [Hu]):

TABLE 2

\begin{tabular}{clc}
\hline Type & \multicolumn{1}{c}{$B(z, \bar{w})$} & $\gamma$ \\
\hline I & $\operatorname{det}\left(I_{m}-z \bar{w}^{\prime}\right)^{-(n+m)}$ & $n+m$ \\
II & $\operatorname{det}\left(I_{n}-z \bar{w}\right)^{-(n+1)}$ & $n+1$ \\
III & $\operatorname{det}\left(I_{n}+z \bar{w}\right)^{-(n-1)}$ & $n-1$ \\
IV & $\left(1+\left|z w^{\prime}\right|^{2}-2 z \bar{w}^{\prime}\right)^{-n}$ & $n$ \\
\hline
\end{tabular}

We recall that a $G$-invariant measure on $\Omega$ is given by

$$
B(z, \bar{z}) d z \text {. }
$$

In the following sections we shall use the weight $w_{\Lambda, p, \alpha}(z)(0<p<\infty, \alpha \in R)$ defined by

$$
w_{\Lambda, p, \alpha}(z)=\left|\psi_{\Lambda}(x)\right|^{(1+\alpha) p} B(z, \bar{z}) \quad(z=0 \cdot x)
$$

and for simplicity we put

$$
A_{\Lambda}^{p}(\Omega)=A_{w_{\Lambda, p}, 0}^{p}(\Omega) .
$$

2.4. Let $\Lambda$ be as in 2.2 and suppose that $\langle\Lambda+\rho, \alpha\rangle\langle 0$ for every non compact positive root $\alpha$. Then $A_{A}^{2} \neq\{0\}$ by Lemma $2.1(4)$ and the operator $U_{A}$ on $A_{A}^{2}$ defined by $U_{A}(g) f(x)=f(x g)\left(f \in A_{A}^{2}, g \in G\right.$ and $\left.x \in B G\right)$ preserves the $A_{A}^{2}$-norm of $f$. Then $\left(U_{A}, A_{A}^{2}\right)$ is a continuous irreducible unitary representation of $G$ whose matrix coefficients are square integrable on $G$, what is called the holomorphic discrete series of $G$ (cf. [Kn], p. 231). As a representation of the compact group $K$, the space $A_{A}^{2}$ is decomposed into irreducible components which we denote by $V_{A_{i}}(i \in N)$, so we choose a complete orthonormal system $\phi_{j}^{i}=\phi_{\Lambda_{j}}^{i}\left(1 \leqq j \leqq d_{i}=\operatorname{dim} V_{A_{i}}, i \in N\right)$ of $A_{A}^{2}$ such that $\phi_{j}^{i} \in V_{\Lambda_{i}}\left(1 \leqq j \leqq d_{i}\right)$ and we may assume that $\Lambda_{1}=\Lambda$ and $\phi_{1}^{1}=c_{\Lambda}^{-1} \psi_{\Lambda}$. Then the matrix coefficient of $U_{A}$ is given by 


$$
U_{j j^{\prime}}^{i j^{\prime}}(x)=U_{A j j^{\prime}}^{i j^{\prime}}(x)=\left(U_{A}(x) \phi_{j^{\prime}}^{i \prime^{\prime}}, \phi_{j}^{i}\right) \quad(x \in G) .
$$

Since $U_{A}(x)$ is a unitary operator on $A_{A}^{2}$, for fixed $j^{\prime}, i^{\prime}$ and $x$, each $U_{j j^{\prime}}^{i \prime}(x)$ is a Fourier coefficient of the $L^{2}$ function $U_{A}(x) \phi_{j}^{\prime \prime}$, on $G$, and thus it follows that

$$
\sum_{i, j}\left|U_{j j^{\prime}}^{i \prime^{\prime}}(x)\right|^{2}<\infty
$$

For simplicity we put

$$
U_{j}^{i}=U_{1 j}^{1 i}
$$

Then $U_{1}^{1}=\psi_{A}$ (cf. [Kn], p. 236). Basic properties of these matrix coefficients are summarized in the following two lemmas.

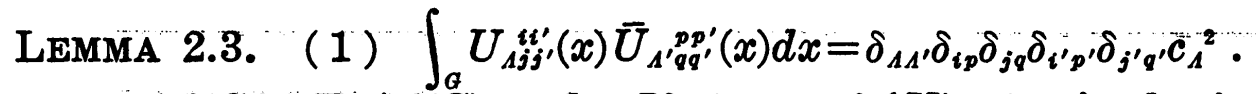

(2) $A_{A}{ }^{2}(G)=E_{A} A_{A}{ }^{2}(G)=$ the $L^{2}$ span of $\left\{U_{j}^{i} ; 1 \leqq j \leqq d_{i}, i \in N\right\}$.

(3) $E_{A^{\prime}} U_{A j j^{\prime}}^{i j^{\prime}}=\delta_{A A^{\prime}} \delta_{1 i} U_{A^{\prime j} j^{\prime}}^{11}$.

(4) $U_{j}^{i}=c_{1} \phi_{j}^{i}$.

Proof. (1) follows from general theory of discrete series (cf. [Va], p. 437) and the rest are obvious from (1) and definition. Q.E.D.

LEMMA 2.4. (1) $U_{j j^{\prime}}^{i \prime^{\prime}}\left(x y^{-1}\right)=\sum_{p, q} U_{j q}^{i p}(x) \bar{U}_{j^{\prime} q}^{i, p}(y)(x, y \in G)$.

(2) $U_{A j j^{\prime}, *}^{i i^{\prime}} U_{A^{\prime} q q^{\prime}}^{p p^{\prime}}=\delta_{A A^{\prime}} \delta_{i^{\prime} p} \delta_{j^{\prime} q} c_{A}{ }^{2} U_{A j q^{\prime}}^{i, 0}$

(3) $f=c_{A}^{-2} \psi_{A}^{*} f$ for $f \in A_{A}^{2}(G)$.

Proof. (1) follows from (2.13) and (2.14). Especially, by the Schwarz inequality the sum of the right hand side is absolutely convergent. Therefore, since $\sum_{p, q}\left|\left(f, U_{j q}^{i p}\right)\right|^{2}<\infty$ for $f \in L^{2}(G)$ by the Plancherel theorem on $G$, (2) follows from (1) and Lemma 2.3 (1), and moreover (3) is obvious for a finite linear combination of $U_{j}^{i}$. Now let $f$ be an arbitrary function in $A_{A}^{2}(G)$. Since $\psi_{A^{*}} * f$ is a continuous function on $G$ and $\left\|\psi_{A^{*}} f\right\|_{\infty} \leqq$ $\left\|\psi_{1}\right\|_{2}\|f\|_{2}<\infty$, (3) is also valid from Lemma $2.3(2)$ and the argument approximating $f$ with a finite linear combination of $U_{j}^{i}$.

Q.E.D.

\section{§3. Estimate for matrix coefficients.}

Throughout the rest of the paper we assume that

Assumption. $\Omega$ is one of the classical bounded symmetric domains in Table 1 and $\tau_{A}$ (see 2.2) is one dimensional representation of $K$.

It is known that the dominant integral forms on $\mathfrak{h}_{c}$ which correspond 
to one dimensional representations of $K$ are parametrized by integers, say $\Lambda_{l}(l \in Z)$. Explicitly $\tau_{\Lambda_{l}}$ is given as follows (cf. [Mu]):

TABLE 3

\begin{tabular}{lll}
\hline Type & \multicolumn{1}{c}{$k \in K$} & \multicolumn{1}{c}{$\tau \Lambda_{l}(k)$} \\
\hline I & {$\left[\begin{array}{rr}U & V\end{array}\right]$} & $(\operatorname{det} V)^{\imath}$ \\
II & {$\left[\begin{array}{rr}U & V \\
-V & U\end{array}\right]$} & $(\operatorname{det}(U+i V))^{-\imath}$ \\
III & {$\left[\begin{array}{rr}U & V \\
-V & U\end{array}\right]$} & $(\operatorname{det}(U+i V))^{-(1 / 2) \imath}$ \\
IV & {$\left[\begin{array}{rrr}U & \\
\cos \theta & \sin \theta \\
-\sin \theta & \cos \theta\end{array}\right]$} & $e^{i l \theta}$ \\
\hline
\end{tabular}

3.1. Under the assumption that $\operatorname{dim} \tau_{A_{l}}=1$ the relation between $\psi_{A_{l}}$ and $B(z, \bar{z})$ is given by the following

LEMMA 3.1.

$$
\left|\psi_{A_{l}}(g)\right|=B(z, \bar{z})^{-l / 2 \gamma} \quad(z=0 \cdot g),
$$

where $\gamma$ is the number listed in Table 2.

Proof. We choose positive non compact roots $\gamma_{1}, \gamma_{2}, \cdots, \gamma_{8}$ and the root vectors $X_{r_{i}}(1 \leqq i \leqq s)$ such that $\sum_{i} R\left(X_{r_{i}}+X_{-r_{i}}\right)$ is a maximal abelian subspace of $\mathfrak{p}$, where $\mathfrak{g}=\mathfrak{f}+\mathfrak{p}$ is a Cartan decomposition of $\mathfrak{g}$ (cf. [Kn], p. 228). Then each element $g \in G$ has a decomposition that $g=$ $k^{\prime} \exp \left(\sum_{i} t_{i}\left(X_{r_{i}}+X_{-r_{i}}\right)\right) k \quad\left(k, k^{\prime} \in K \quad\right.$ and $\left.t_{i} \geqq 0\right)$. Since $\psi_{\Lambda_{l}}(g)=\tau_{\Lambda_{l}}(\mu(g))$ $\left(g \in P^{-} K_{c} P^{+}\right)$by the definition (2.4), it easily follows from Corollary in [Kn], p. 229 that

$$
\left|\psi_{A_{l}}(g)\right|=\left(\operatorname{ch} t_{1} \operatorname{ch} t_{2} \cdots \operatorname{ch} t_{s}\right)^{-l} .
$$

On the other hand, $z=0 \cdot g=\zeta \cdot k$, where $\zeta=0 \cdot \exp \left(\sum_{i} t_{i}\left(X_{r_{i}}+X_{-r_{i}}\right)\right)$. Therefore, by substituting for $z$ in $B(z, \bar{z})$ given in Table 2 , we can deduce the desired equality.

Q.E.D.

Now we shall obtain a condition of $l$ for which $\psi_{A_{l}}$ belongs to $L^{p}(G)$. Since a $G$-invariant measure on $\Omega$ is given by $B(z, \bar{z}) d z$, fixed from now on, it follows from Lemma 3.1 that

$$
\left\|\psi_{A_{l}}\right\|_{p}^{p}=\int_{\Omega} B(z, \bar{z})^{-l_{p} / 2 \gamma+1} d z
$$

This type of integrals is calculated in [Hu], Theorems 2.2.1, 2.3.1, 2.4.1 and 2.5.1. So, we can decide the $L^{2}$ and $L^{p}$ conditions for $\psi_{A_{l}}$ as in 
Table 4. Especially, the $L^{2}$ condition implies that $\left(U_{A_{l}}, A_{A_{l}}^{2}\right)$ (see 2.4) corresponds to a holomorphic discrete series of $G$. If $\psi_{A_{l}}$ does not belong to $L^{1}(G)$, we can find the least number $\alpha_{A_{l}}>0$ satisfying

$$
\left|\psi_{A_{l}}\right|^{1+\alpha} \in L^{1}(G) \quad \text { for all } \alpha>\alpha_{\Lambda_{l}} \text {. }
$$

TABLE 4

\begin{tabular}{clll}
\hline Type & $L^{2}$-condition & \multicolumn{1}{c}{$L^{p}$-condition } & $\alpha_{\Lambda_{l}}$ \\
\hline I & $l>n+m-1$ & $l>2(n+m-1) / p$ & $2(n+m-1) / l-1$ \\
II & $l>n$ & $l>2 n / p$ & $2 n / l-1$ \\
III & $l>n-3 / 2$ & $l>(2 n-3) / p$ & $(2 n-3) / l-1$ \\
IV & $l>n-1$ & $l>2(n-1) / p$ & $2(n-1) / l-1$ \\
\hline
\end{tabular}

REMARK 3.2. (1) Except type III the $L^{2}, L^{p}$-conditions and $\alpha_{A_{l}}$ are given by $l>\gamma-1, l>2(\gamma-1) / p$ and $2(\gamma-1) / l-1$ respectively.

(2) If $\psi_{A_{l}}$ is in $L^{2}(G)$ but not in $L^{1}(G)$, then $0 \leqq \alpha_{A_{l}}<1$.

3.2. We shall define an intertwining operator between $A_{\Lambda}^{2}$ and $A_{\Lambda}^{2}(\Omega)$ when these spaces don't vanish. So, we assume that $\Lambda=\Lambda_{l}$ satisfies the $L^{2}$-condition (see Lemma 2.1(4) and Table 4). For a complex valued function $f$ on $B G$ satisfying $E_{A} f=f$ we define

$$
I_{\Lambda}(f)(z)=\psi_{\Lambda}(g)^{-1} f(g) \quad(z=0 \cdot g),
$$

and for a complex valued function $F$ on $\Omega$ we define

$$
\left(T_{\Lambda}(g) F\right)(z)=\psi_{\Lambda}(x)^{-1} \psi_{\Lambda}(x g) F(z \cdot g)
$$

where $g, x \in G$ and $z=0 \cdot x$. Under the assumption that $\operatorname{dim} \tau_{A}=1$ these definitions are well-defined, and it easily follows that

Lemma 3.3. $\left(U_{A}, A_{A}^{2}\right)$ and $\left(T_{A}, A_{A}^{2}(\Omega)\right)$ are unitary equivalent and $I_{A}$ is an intertwining operator of $A_{\Lambda}^{2}$ onto $A_{\Lambda}^{2}(\Omega)$.

We put

$$
\psi_{j}^{i}=\psi_{A_{j}^{i}}^{i}=I_{\Lambda}\left(\phi_{j}^{i}\right) \quad\left(1 \leqq j \leqq d_{i}, i \in N\right) .
$$

Then, $\left\{\psi_{j}^{i}\right\}$ is a complete orthonormal system in $A_{\Lambda}^{2}(\Omega)$ and, as considered in [Hu] (see Table 5 below), we may assume that each $\psi_{j}^{i}$ is a homogeneous polynomial on $\Omega$ whose degree depends on $i$. Therefore, by (3.3), (3.5) and Lemma 2.3(4) we can find a constant $c_{i}$, which only depends on $i$, such that

$$
\left|U_{A^{i}}^{i}\right| \leqq c_{i}\left|\psi_{A}\right| \quad \text { for all } 1 \leqq j \leqq d_{i}
$$


Now we shall obtain a similar estimate for $U_{A j j^{\prime}}^{i \prime^{\prime}}$. The following first two Lemmas obtained by [CR], Lemmas 2.2 and 2.8 are important estimates we will need for the Bergman kernel, where the constant $\varepsilon_{D}$ in [CR] corresponds to $1 / \gamma$ in our paper.

LEMma 3.4 ([CR]). If $\alpha>r>-1 / \gamma$, then

$$
\int_{\Omega}|B(z, \bar{\zeta})|^{1+\alpha} B(\zeta, \bar{\zeta})^{-r} d \zeta \leqq C_{\alpha, r} B(z, \bar{z})^{\alpha-r} \quad(z \in \Omega),
$$

where $C_{\alpha, r}$ does not depend on $z$.

LEMMA 3.5 ([CR]). Let us suppose that $p>1, r>-1 / \gamma, \theta>(p-2)(r+1)$ and $\alpha=2 r+2+\theta$. Then the operator $T$ which sends the function $F$ defined on $\Omega$ to the new function $T F$ on $\Omega$ defined by

$$
(T F)(z)=\int_{\Omega} F(\zeta)|B(z, \bar{\zeta})|^{\alpha / p} B(\zeta, \bar{\zeta})^{\alpha / p+1} d \zeta \quad(z \in \Omega)
$$

is a bounded operator of $L^{p}\left(\Omega, B(\zeta, \bar{\zeta})^{-r} d \zeta\right)$ into itself.

LEMMA 3.6.

$$
\psi_{A}\left(x y^{-1}\right)=c_{A}{ }^{2} \psi_{A}(x) \bar{\psi}_{A}(y) B(z, \bar{\zeta})^{l / r}
$$

where $x, y \in G, z=0 \cdot x$ and $\zeta=0 \cdot y$.

Proof. For $f$ in $A_{A}^{2}(G)$ it follows from Lemma 2.4 (3) that

$$
\psi_{\Lambda}(x)^{-1} f(y)=c_{A}^{-2} \int_{G}\left[\psi_{\Lambda}(x)^{-1} \psi_{\Lambda}\left(x y^{-1}\right) \psi_{\Lambda}(y)\right] \psi_{A}(y)^{-1} f(y) d y .
$$

By Lemmas 3.3 and 3.1 this means that for $F$ in $A_{\Lambda}^{2}(\Omega)$

$$
F(z)=c_{A}^{-2} \int_{\Omega}\left[\psi_{A}(x)^{-1} \psi_{A}\left(x y^{-1}\right) \bar{\psi}_{A}(y)^{-1}\right] F(\zeta) B(\zeta, \bar{\zeta})^{-l / r+1} d \zeta,
$$

where $z=0 \cdot x$ and $\zeta=0 \cdot y$. Then noting the form of $[\cdots]$ and comparing the reproducing formula in [CR], Lemma 2.1, we can obtain the desired relation.

LeMma 3.7. For each $\varepsilon>0$

$$
\sup _{x \in G}\left|\psi_{A}(x)\right|^{-1+\varepsilon} \int_{G}\left|\psi_{A}\left(x y^{-1}\right)\right|\left|\psi_{A}(y)\right| d y<\infty .
$$

Proof. By Lemmas 3.1 and 3.6 the assertion is equivalent to 


$$
\sup _{x \in \bar{G}} B(z, \bar{z})^{-\alpha} \int_{\Omega}|B(z, \bar{\zeta})|^{\beta} B(\zeta, \bar{\zeta})^{-\beta+1} d \zeta<\infty,
$$

where $\beta=l / \gamma$ and $\alpha>0$. We recall that $l>\gamma-1$ and, since $\left|\psi_{A}\right| \leqq 1$, $B(z, \bar{z})=\left|\psi_{1}\right|^{-2 \gamma / l} \geqq 1$. Therefore, replacing $B(\zeta, \bar{\zeta})^{-\beta+1}$ in the integrand by $B(\zeta, \bar{\zeta})^{-\beta+\delta+1}(0<\delta<\alpha)$, we can obtain the desired finiteness from Lemma 3.4.

Q.E.D.

Then we see that

COROLlaRY 3.8. For each $i, i^{\prime} \in N$ and $\varepsilon>0$ there exists a constant $c_{i i^{\prime}}$ such that

$$
\left|U_{i j^{\prime} j^{\prime}}^{i j^{\prime}}\right| \leqq c_{i i^{\prime}}\left|\psi_{A}\right|^{1-i} \quad \text { for } \quad 1 \leqq j \leqq d_{i}, 1 \leqq j^{\prime} \leqq d_{i^{\prime}} .
$$

PRoof. It follows from Lemmas 2.4(2), 2.1(3) and (3.6) that $\left|U_{A j j^{i j}}^{i j^{\prime}}\right| \leqq$ $c_{A}^{-2}\left|U_{A j 1}^{i 1}\right| *\left|U_{A 1 j^{\prime}}^{1 i^{\prime}}\right| \leqq c_{A}{ }^{-2} c_{i} c_{i^{\prime}}\left|\psi_{A}\right| *\left|\psi_{A}\right|$. Therefore, Lemma 3.7 implies the desired inequality.

3.3. Let $(\Lambda, p)$ be a pair of a dominant integral form on $\mathfrak{h}_{c}$ and $1 \leqq p \leqq 2$. We say that $(\Lambda, p)$ or $\left(\psi_{A}, p\right)$ is regular if $(\Lambda, p)$ satisfies the condition that

$$
\psi_{A} \in L^{p}(G)
$$

(see Table 4). Then the condition ( $R$ ) is equivalent to the one of the following three conditions:

$$
\begin{array}{lll}
\psi_{A} \in L^{p}(G) & \text { and } & 1<p<2 \\
\psi_{A} \in L^{1}(G) & \text { and } & p=1 \\
\psi_{1} \in L^{2}(G) & \text { and } & p=2 .
\end{array}
$$

If $(\Lambda, p)$ is regular, each $U_{j}^{i}$ belongs to $L^{p}(G)$ by (3.6) and then it satisfies the norm equality:

$$
\left\|U_{j}^{i}\right\|_{p}=c_{A}\left\|\psi_{j}^{i}\right\|_{p, w_{A, p, 0}}
$$

(see Lemma 2.3(4), Lemma 3.1, (2.10), (2.11), (3.3) and (3.5)). Here we note that

$$
A_{A}^{p}(\Omega) \text { is the }\|\cdot\|_{p, W_{A, p, 0}} \text { norm span of }\left\{\psi_{j}^{t}\right\} \text {. }
$$

In fact, $A_{\Lambda}^{p}(\Omega)$ contains densely all functions holomorphic in a domain bigger than $\Omega$ and they can be approximated by a finite linear combination of $\left\{\psi_{i}^{i}\right\}$ (cf. the proof of [DRS], Theorem 3). Therefore, it follows that 
Proposition 3.9. Let us suppose that $(\Lambda, p)$ is regular.

(1) $A_{A}^{p}(G)=E_{A} A_{A}^{p}(G)=$ the $L^{p}$-span of $\left\{U_{A j}^{i} ; 1 \leqq j \leqq d_{i}, i \in N\right\}$.

(2) $\left(U_{A}, A_{A}^{p}\right)$ and $\left(T_{A}, A_{A}^{p}(\Omega)\right)$ are equivalent as Banach space representation and $I_{A}$ is the norm preserving intertwining operator of $A_{A}^{p}(G)$ and $A_{A}^{p}(\Omega)$.

We recall that

$$
\text { if }(\Lambda, p) \text { is regular, then } p>2(\gamma-1) / l \text {. }
$$

Actually, except Type III, the condition $p>2(\gamma-1) / l$ is equivalent to the $L^{p}$ condition (R) and when $\Omega$ is of Type III, it is weaker than (R) (see Remark 3.2 and Table 4).

Moreover, since $\left|U_{A j j^{\prime}}^{i \prime^{\prime}}\right| \leqq c_{A}^{-2} c_{i} c_{i^{\prime}}\left|\psi_{A}\right| *\left|\psi_{A}\right|$ (see the proof of Corollary 3.8), we see that

$$
\text { if }(\Lambda, p) \text { satisfies (R1), then }\left\|U_{\Lambda j^{\prime} j^{\prime}}^{i{ }^{\prime}}\right\|_{1} \leqq c \text {, }
$$

where $c=c_{A}^{-2} c_{i} c_{i^{\prime}}\left\|\psi_{A}\right\|_{1}^{2}$, which does not depend on $j$ and $j^{\prime}$.

Proposition 3.10. If $(\Lambda, p)$ is regular, then for each $i, i^{\prime} \in N$ there exists a constant $C_{i i}$, such that

$$
\left\|U_{A j^{\prime} j^{\prime} *}^{i \prime^{\prime}} f\right\|_{p} \leqq C_{i i^{\prime}}\|f\|_{p} \quad \text { for all } f \in L^{p}(G) \text { and } j, j^{\prime} \text {. }
$$

Proof. First let $(\Lambda, p)$ satisfy (R2). Then it is enough to show the inequality for $f \in C_{c, F}^{\infty}(G)$, the set of all compactly supported $C^{\infty}$ functions on $G$ with finite $K$-types, because $C_{c, F}^{\infty}$ is dense in $L^{2}(G)$.

Let $\phi$ be an elementary spherical function on $G$ (cf. [Va], Part II, §8). Then for all $\varepsilon>0$ it belongs to $L^{2+\varepsilon}(G)$ (cf. [Va], Theorem 30). On the other hand, by Corollary 3.8 and Table $4, U_{A j^{\prime}}^{i j^{\prime}}$ belongs to $L^{2-\delta}(G)$ for a sufficiently small $\delta>0$. Therefore, $\left(\phi, U_{A j j^{\prime}}^{i i^{\prime}}\right)$ is well defined, and it must be equal to 0 , because both the functions are eigenfunctions of the center of the universal enveloping algebra of $\mathfrak{g}_{c}$ with different eigenvalues. Then noting the Plancherel theorem for $f \in C_{c, F}^{\infty}$ (see [HC2]), we see that the operator $U_{A j j^{\prime}}^{i j^{\prime}} *$ vanishes the finite wave packets of $f$. Moreover, by the same argument, it vanishes a finite linear combination of the matrix coefficients of discrete series different from $U_{A}$. Thus, it follows that

$$
U_{\Lambda j^{\prime}}^{i j^{\prime} *} f=U_{A j j^{\prime} * *^{\circ}}^{i{ }^{\prime}} f_{A},
$$

where ${ }^{\circ} f_{A}=c_{A}^{-1} \sum_{p, q, p^{\prime}, q^{\prime}}\left(f, U_{A q q^{\prime}}^{p p \prime^{\prime}}\right) U_{A q q^{\prime}}^{p p^{\prime}}$ (the sum is finite). Then it follows from Lemma $2.4(2)$ that

$$
U_{A j j^{\prime}}^{i i^{\prime} *} f=c_{A} \sum_{p^{\prime}, q^{\prime}}\left(f, U_{A j q^{\prime}}^{i p^{\prime}}\right) U_{A j q^{\prime}}^{i p^{\prime}}
$$


and from the Perseval equality for $f$ that

$$
\left\|U_{A j j^{\prime}}^{i i^{\prime}} * f\right\|_{2}^{2}=c_{\Lambda} \sum_{p^{\prime}, q^{\prime}} \mid\left(f,\left.U_{h j q^{\prime}}^{i p)^{\prime}}\right|^{2} \leqq c_{A}{ }^{2}\|f\|_{2}^{2} .\right.
$$

If $(\Lambda, p)$ satisfies (R1), then the desired inequality is obvious from (3.11).

Now let us suppose that $(\Lambda, p)$ satisfies (R0). By (3.10) we can choose an $\varepsilon>0$ such that $p>2(\gamma-1) / l(1-\varepsilon)$. Then by Corollary 3.8 we

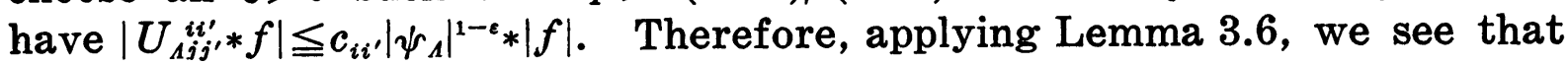

$$
\begin{aligned}
& \left|U_{A j j^{\prime} * *}^{i i^{\prime} *} f(x)\right|\left|\psi_{\Lambda}(x)\right|^{-(1-\varepsilon)} \\
& \leqq c_{i i^{\prime}} c_{\Lambda}{ }^{2(1-\varepsilon)} \int_{\Omega}\left[|f|_{K}(g)\left|\psi_{\Lambda}(g)\right|^{-(1-\varepsilon)}\right] \\
& \quad \times|B(z, \bar{\zeta})|^{(1-8) l / r} B(\zeta, \bar{\zeta})^{-(1-\varepsilon) l / \tau+1} d \zeta,
\end{aligned}
$$

where $z=0 \cdot x, \quad \zeta=0 \cdot g$ and $|f|_{K}(g)=\int_{K}|f(k g)| d k$. Here we note that $|f|\left|\psi_{A}\right|^{-(1-\varepsilon)}$ belongs to $L^{p}\left(\Omega, B(\zeta, \bar{\zeta})^{-r} d \zeta\right)_{K}$ for $r=(1-\varepsilon) l p / 2 \gamma-1$. Therefore, applying Lemma 3.5 as $\theta=0$ and $r=(1-\varepsilon) l p / 2 \gamma-1$, we see that the left hand side of the above inequality also belongs to the $B(\zeta, \bar{\zeta})^{-r}$-weighted $L^{p}$ space on $\Omega$, in particular, $U_{A j j^{\prime}, * f}^{i j^{\prime}}$ belongs to $L^{p}(G)$ (see Lemma 3.1).

Q.E.D.

REMARK 3.11. The case of $G=S U(1,1)$ and $\Omega=D$ we can obtain the estimate in Proposition 3.10 by using the Kunze-Stein phenomenon (see [CS] and [Co]). In our general case the phenomenon is also applicable to obtain the $L^{p}$ estimate, however we have to replace the condition (R0) with the following (R0)':

(R0)' $\quad 1<p<2$ and $\psi_{1} \in L^{q}(G)$ for all $q>1$

(see [CS]). Although this condition is equivalent to (R0) in the case of $S U(1,1)$, generally, it is stronger than (R0).

\section{§4. Fourier transform associated with $T_{4}$ •}

We retain the notation and assumption in the previous section.

Before giving the definition of the Fourier transform associated with $T_{A}$ we shall give a sense of the following equation (4.1). Let $f$ be in $L^{p}(G)$ and $1 \leqq p \leqq 2$. Since $U_{l n}^{k m}$ is in $L^{q}(G)$ for all $q \geqq 2$ by (3.1) and Corollary 3.8, $U_{l n}^{k m} * f$ is well-defined. Then we can obtain that

$$
\begin{aligned}
U_{j l}^{i k} *\left(U_{l n}^{k m} * f\right) & =\left(U_{j l}^{i k} * U_{l n}^{k m}\right) * f \\
& =c_{A}{ }^{2} U_{j n}^{i m} * f
\end{aligned}
$$


Here the second equation follows from Lemma 2.4 (2), and the first one is guaranteed by the Fubini theorem. Actually, when $p=2$,

$$
\begin{aligned}
\left\|U_{j l}^{i k} *\left(U_{l n}^{k m} * f\right)\right\|_{\infty} & \leqq\left\|U_{j l}^{i k}\right\|_{2}\left\|U_{l n}^{k m} * f\right\|_{2} \\
& \leqq c_{A}{ }^{3}\|f\|_{2}<\infty
\end{aligned}
$$

by Lemma 2.3 (1) and Proposition 3.10, and when $1 \leqq p<2$,

$$
\begin{aligned}
\left\|U_{j l}^{i k} *\left(U_{l n}^{k m} * f\right)\right\|_{\infty} & \leqq\left\|U_{j l}^{i k}\right\|_{2}\left\|U_{l n}^{k m} * f\right\|_{2} \\
& \leqq c c_{A}{ }^{2}\|f\|_{p}<\infty
\end{aligned}
$$

by the Kunze-Stein phenomenon (see [Co]). Especially, $U_{l n}^{k m} * f$ is an $L^{2}$ function on $G$.

Let $\Lambda=\Lambda_{l}$ be a dominant integral form on $\mathfrak{h}_{c}$, which corresponds to one dimensional representation $\tau_{A_{l}}$ of $K$ (see Table 3 ), and suppose that $\Lambda$ satisfies the $L^{2}$-condition in Table 4 . Then $\left(T_{\Lambda}, A_{\Lambda}^{2}(\Omega)\right)$ is a holomorphic discrete series of $G$. For $f$ in $L^{p}(G)(1 \leqq p \leqq 2)$ we define the Fourier transform $F_{A j}^{i}(f)\left(1 \leqq j \leqq d_{i}, i \in N\right)$ as follows:

$$
\begin{aligned}
F_{A j}^{i}(f)(z) & =\int_{G} f(g) T_{A}\left(g^{-1}\right) \psi_{A j}^{i}(z) d g \quad(z \in \Omega) \\
& =I_{A}\left(\phi_{j}^{i} * f\right)(z) .
\end{aligned}
$$

Moreover, we define

$$
P_{A j}^{i}(f)=c_{A}^{-2} U_{A j j}^{i i} * f,
$$

and call it a "discrete part" of $f$. As pointed in the equation (4.1), these convolution operators are well-defined on $L^{p}(G)(1 \leqq p \leqq 2)$ and the discrete part is an $L^{2}$ function on $G$. For simplicity we put

$$
F_{A}=F_{A 1}^{1} \text { and } P_{A}=P_{A 1}^{1}
$$

Now, applying (4.1) and Lemma $2.4(2)$, we see that

$$
\begin{aligned}
& U_{j j}^{i i} *\left(U_{j j}^{i i} * f\right)=c_{A}{ }^{2}\left(U_{j j}^{i i} * f\right), \\
& U_{1 j}^{1 i} *\left(U_{j j}^{i i} * f\right)=c_{A}{ }^{2}\left(U_{1 j}^{1 i} * f\right), \\
& U_{j 1}^{i 1} *\left(U_{1 j}^{11} * f\right)=c_{A}{ }^{2}\left(U_{j j}^{i i} * f\right) ;
\end{aligned}
$$

so, $U_{1 j}^{1 i} *\left(U_{j j}^{i i} * f\right)=0$ means that $0=U_{j 1}^{i 1} *\left(U_{1 j}^{1 i} * f\right)=c_{A}{ }^{2} U_{j j}^{i i} * f$. Therefore, $P_{A j}^{i}$ is a projection operator which maps $f \in L^{p}(G)$ to the discrete part of $f$ and moreover,

$$
\begin{aligned}
& F_{A j}^{i}\left(L^{p}(G)\right)=F_{A j}^{i}\left(P_{A j}^{i}\left(L^{p}(G)\right)\right) \text { and } \\
& F_{A j}^{i} \text { is injective on } P_{A j}^{i}\left(L^{p}(G)\right) .
\end{aligned}
$$


Especially, Propositions 3.10 and 3.9 (1) imply that

$$
\begin{aligned}
& \text { if }(\Lambda, p) \text { is regular, } P_{\Lambda j}^{i}\left(L^{p}(G)\right) \subset L^{p}(G) \text { and } \\
& P_{\Lambda}\left(L^{p}(G)\right)=A_{\Lambda}^{p}(G) \text {. }
\end{aligned}
$$

Our problem can be stated as follows.

ProbLEM. Give a characterization of $F_{A j}^{i}\left(L^{p}(G)\right)(1 \leqq p \leqq 2)$ as a space of holomorphic functions on $\Omega$.

The first result for $F_{A j}^{i}\left(L^{p}(G)\right)$ is the independence of $i$ and $j$.

THEOREM 4.1. Let $1 \leqq p \leqq 2$ and $\Lambda=\Lambda_{l}$ satisfy the $L^{2}$-condition. Then

$$
F_{A j}^{i}\left(L^{p}(G)\right)=F_{\Lambda}\left(L^{p}(G)\right) \text { for all } 1 \leqq j \leqq d_{i}, i \in N \text {. }
$$

Proof. By (4.1), (4.2) and (4.6) it is enough to prove that the correspondence of $f$ and $f^{\sim}=U_{j}^{i} * f$ gives a bijection between $P_{\Lambda j}^{i}\left(L^{p}(G)\right.$ ) and $P_{\Lambda}\left(L^{p}(G)\right)$.

The case of $(\Lambda, p)$ is regular: it follows from Proposition 3.9 and (4.7) that $f \in P_{A j}^{i}\left(L^{p}(G)\right)$ belongs to $L^{p}(G)$. Then, by Proposition $3.10 f^{\sim}$ also belongs to $L^{p}(G)$. Since $P_{A}\left(f^{\sim}\right)=f^{\sim}$ by (4.1), $f^{\sim}$ belongs to $P_{A}\left(L^{p}(G)\right)$. Clearly, this argument is reversible because of $f=c_{A}^{-2} U_{j 1}^{i 1} * f^{\sim}$. Then we can obtain the desired bijection.

The case of $(\Lambda, p)$ is non regular: first we shall prove a lemma which will play an important role in $\S 5$.

LEMMA 4.2. For each $U_{j j^{\prime}}^{i i^{\prime}}=U_{A j j^{\prime}}^{i i^{\prime}}\left(\Lambda=\Lambda_{l}\right)$ and $\alpha>\alpha_{\Lambda}$ we put

$$
\left[U_{j j^{i}}^{i j^{\prime}}\right](g)=C\left|\psi_{\Lambda}(g)\right|^{\alpha} U_{j j^{\prime}}^{i \prime^{\prime}}(g) \quad(g \in G),
$$

where the constant $C=C\left(\alpha ; i, j ; i^{\prime}, j^{\prime}\right)$ is given by

$$
C \int_{G}\left|\psi_{\Lambda}(g)\right|^{\alpha}\left|U_{j j^{\prime}}^{u \prime}(g)\right|^{2} d g=c_{A}{ }^{2} .
$$

Then $\left[U_{j j^{i}}^{i{ }^{\prime}}\right]$ is an $L^{1}$ function on $G$ such that

$$
U_{j j^{\prime}}^{i i^{\prime}}=P_{A j}^{i}\left(\left[U_{j j^{\prime}}^{\left.\left.i i^{\prime}\right]\right)}\right.\right. \text {. }
$$

Proof. We take an $\varepsilon>0$ such that $\alpha-\varepsilon>\alpha_{\Lambda}$. Then by Corollary 3.8 it follows that

$$
\left[U_{j j^{\prime}}^{i i^{\prime}}\right] \leqq c_{i i^{\prime}}\left|\psi_{A}\right|^{\alpha}\left|\psi_{A}\right|^{1-\varepsilon}=c_{i i^{\prime}}\left|\psi_{A}\right|^{1+(\alpha-\varepsilon)}
$$

and thus $\left[U_{j j^{\prime \prime}}^{i \prime}\right]$ belongs to $L^{1}(G)$ (see (3.2)). Then, since $U_{j j^{\prime}}^{i \prime} \in L^{2}(G) \cap L^{\infty}(G)$, $U_{j j^{\prime}}^{i t} *\left[U_{j j^{i \prime}}^{i \prime^{\prime}}\right]$ also belongs to $L^{2}(G) \cap L^{\infty}(G)$. Therefore, 


$$
\begin{aligned}
& P_{A j}^{i}\left(\left[U_{j j^{\prime}}^{i \prime^{\prime}}\right]\right)(x)={c_{A}}^{-2} U_{j j^{i j} *}^{i i}\left[U_{j j^{i j}}^{i{ }^{\prime}}\right](x) \\
& =c_{A}^{-2} \int_{G} U_{j j}^{i i}\left(x y^{-1}\right)\left[U_{j j^{\prime}}^{i i^{\prime}}\right](y) d y \\
& =C c_{\Lambda}{ }^{-2} \int_{G} \sum_{p, q} U_{j q}^{i p}(x) \bar{U}_{j q}^{i p}(y)\left|\psi_{\Lambda}(y)\right|^{\alpha} U_{j j^{\prime}}^{i \prime^{\prime}}(y) d y
\end{aligned}
$$

(see Lemma $2.4(1)$ ), and by changing the variable $y$ to $k y k^{\prime}\left(k, k^{\prime} \in K\right)$,

$$
=C c_{\Lambda}^{-2} \int_{a} \int_{K} \int_{K} \sum_{p, q} U_{j q}^{i p}(x) \bar{U}_{j q}^{i p}\left(k y k^{\prime}\right)\left|\psi_{\Lambda}\left(k y k^{\prime}\right)\right|^{\alpha} U_{j j^{\prime}}^{i \prime^{\prime}}\left(k y k^{\prime}\right) d k d k^{\prime} d y .
$$

Here we recall the assumption that $\operatorname{dim} \tau_{\Lambda_{l}}=1$, and so $\left|\psi_{A}\left(k y k^{\prime}\right)\right|=\left|\psi_{A}(y)\right|$. Then the orthogonal relations over $K$ and $K^{\prime}$ deduce that

$$
\begin{aligned}
& =C c_{A}{ }^{-2} U_{j j^{\prime}}^{i j^{\prime}}(x) \int_{G}\left|\psi_{A}(y)\right|\left|U_{j j^{\prime}}^{i j^{\prime}}(y)\right|^{2} d y \\
& =U_{j j^{\prime}}^{i \prime^{\prime}}(x)
\end{aligned}
$$

by the definition of $C$.

REMARK 4.3. For an $L^{p}(1 \leqq p \leqq 2)$ function $f$ on $G$ the notation " $[f]$ " means the class, or a representative of the class, of all functions on $G$ whose discrete parts are the same as the one of $f$.

Now we shall return to the proof of the non regular case. Let us suppose that $f$ is in $P_{A^{i}}^{i}\left(L^{p}(G)\right)$. This means that there exists an $L^{p}$ function $[f]$ on $G$ such that $f=c_{A}^{-2} U_{j j}^{i i} *[f]$, and thus, by $(4.1), f^{\sim}=$ $c_{A}^{-2} U_{j}^{i} *[f]$. Then by Lemma 4.2 we can choose an $L^{1}$ function [ $U_{j}^{i}$ ] on $G$ such that $U_{j}^{i}=c_{A}{ }^{-2} U_{1}^{1} *\left[U_{j}^{i}\right]$. Since $U_{1}^{1}$ belongs to $L^{q}(G)$ for all $q \geqq 2$, it follows that

$$
\begin{aligned}
&\left\|\left(U_{1}^{1} *\left[U_{j}^{i}\right]\right) *[f]\right\|_{\infty} \leqq\left\|U_{1}^{1} *\left[U_{j}^{i}\right]\right\|_{q}\|[f]\|_{p} \\
& \leqq\left\|U_{1}^{1}\right\|_{q}\left\|\left[U_{j}^{i}\right]\right\|_{1}\|[f]\|_{p}<\infty,
\end{aligned}
$$

where $1 / p+1 / q=1$. Therefore, by the Fubini theorem, we see that

$$
f^{\sim}=c_{A}^{-4}\left(U_{1}^{1} *\left[U_{j}^{i}\right]\right) *[f]=c_{A}{ }^{-2} P_{A}\left(\left[U_{j}^{i}\right] *[f]\right) .
$$

Since $\left[U_{j}^{i}\right] *[f]$ belongs to $L^{p}(G)$, the above relation means that $f^{\sim}$ also belongs to $P_{\Lambda}\left(L^{p}(G)\right)$. As before, this argument is reversible, so we can obtain the desired bijection.

The constant $C(\alpha ; 1,1 ; i, j)$ in Lemma 4.2 will play an important role in the following sections. So, by calculating explicitly the integral in the definition, in the next lemma we shall show that the constant 
does not depend on $j$ and the square of $C(\alpha ; 1,1 ; i, j)$ corresponds to $C(2 \alpha ; 1,1 ; i, j)$. In this calculation we need the assumption that $\Omega^{\sim}=G / K$ is one of the classical bounded symmetric domains in Table 1 , because we shall cite the results obtained by [Hu].

LEMMA 4.4. Let notation be as in Lemma 4.2. $C\left(\alpha ; i, j ; i^{\prime}, j^{\prime}\right)$ can be defined for $\alpha>\alpha_{A}-1, C(\alpha ; 1,1 ; i, j)$ does not depend on $1 \leqq j \leqq d_{i}$, and if we say $C_{\alpha}^{i}$, that is,

$$
C_{\alpha}^{t} \int_{G}\left|U_{j}^{t}(x)\right|^{2}\left|\psi_{A}(x)\right|^{\alpha} d x=c_{A}{ }^{2},
$$

then

$$
\left(C_{\alpha}^{i}\right)^{2} \sim C_{2 \alpha}^{i} \quad \text { for all } i \in N,
$$

where " " means that the ratio of the left and the right is bounded below and above by positive constants which do not depend on $i$.

Proof. By (3.2), Corollary 3.8 and Table 4 it easily follows that the integral in the definition of the constant exists for $\alpha>\alpha_{A}-1$. The rest of the assertions will be proved by an explicit calculation.

First we shall replace the complete orthonormal system $\left\{\psi_{j}^{i}\right\}$ in $A_{A}^{2}(\Omega)$ (see (2.12) and (3.5)) by another complete orthogonal system $\left\{\psi_{i}^{\prime i}\right\}$ in $A^{2}(\Omega)=A_{1}^{2}(\Omega)(w \equiv 1$ in $(2.9))$, where each $A^{2}$ norm of $\psi_{j}^{\prime i}$ only depends on the homogeneous degree, so on $i$. This replacement of the basis clearly does not effect the assertion of the independence on $j$ of $C(\alpha ; 1,1 ; i, j)$. On the other hand, it follows from the definition of $C(\alpha ; 1,1 ; i, j)$ that

$$
C(\alpha ; 1,1 ; i, j)^{-2}=\left(c_{A}^{-2} \int_{\Omega}\left|\psi_{j}^{i}(\zeta)\right|^{2}\left|\psi_{A}(x)\right|^{2+\alpha} B(\zeta, \bar{\zeta}) d \zeta\right)^{2}
$$

and

$$
\begin{aligned}
C(2 \alpha ; 1,1 ; i, j)^{-1}= & c_{A}^{-2} \int_{\Omega}\left|\psi_{j}^{i}(\zeta)\right|^{2}\left|\psi_{A}(x)\right|^{2+2 \alpha} B(\zeta, \bar{\zeta}) d \zeta \\
= & c_{A}^{-2} \int_{\Omega}\left|\psi_{j}^{i}(\zeta)\right|^{2}\left|\psi_{A}(x)\right|^{2+2 \alpha} B(\zeta, \bar{\zeta}) d \zeta \\
& \times \int_{\Omega}\left|\psi_{j}^{i}(\zeta)\right|^{2}\left|\psi_{A}(x)\right|^{2} B(\zeta, \bar{\zeta}) d \zeta
\end{aligned}
$$

where $\zeta=0 \cdot x$. The last integral is equal to one since each $\psi_{j}^{i}$ is a normalized base in $A_{A}^{2}(\Omega)$ (see (3.5) and (2.12)). Here we note that the assertion (4.9) is equivalent to the " $\sim$ " relation of the right hand sides 
of (1) and (2) and moreover, it is invariant under a scalar multiplication: $\psi_{j}^{i} \rightarrow c \psi_{j}^{i}$. Therefore, if we regard (4.9) as the " " relation of the right hand sides of (1) and (2), the replacement of the basis also does not effect the assertion of (4.9).

Actually, such a system $\left\{\psi^{\prime \prime}{ }_{j}\right\}$ of $A^{2}(\Omega)$ is given by homogeneous polynomials on $\Omega$, which are denoted by $\psi_{f}^{(i)}$ in [Hu], p. 100, p. 131, p. 138 and p. 147 for Type I, II, III and IV respectively.

TABLE 5

\begin{tabular}{clc}
\hline Type & C.O.S. & Index \\
\hline I & $\psi_{f}{ }^{(i)}$ & $1 \leqq i \leqq N\left(f_{1}, \cdots, f_{m}\right) N\left(f_{1}, \cdots, f_{m}, 0, \cdots, 0\right)$ \\
II & $\psi_{f}{ }^{(i)}$ & $f=\left(f_{1}, \cdots, f_{m}\right)$ \\
& & $1 \leqq i \leqq N\left(2 f_{1}, \cdots, 2 f_{n}\right)$ \\
III & $\psi_{f}^{(i)}$ & $f=\left(f_{1}, \cdots, f_{n}\right)$ \\
IV & $1 \leqq i \leqq N\left(f_{1}, f_{1}, \cdots, f_{n}, f_{n}\right)$ \\
& $\left(z z^{\prime}\right)^{i} \psi_{f-2 l}{ }^{(i)}$ & $f=\left(f_{1}, \cdots, f[n / 2]\right)$ \\
\end{tabular}

Roughly speaking, " $f$ " implies the homogeneous type of $\psi_{f}^{(i)}$, that is " $f$ " and " $i$ " correspond respectively to $i$ and $j$ in our notation. The exact definitions of $\psi_{f}^{(i)}, N\left(f_{1}, \cdots, f_{n}\right)$ and $N_{f}$ are given in [Hu], 5.1. Fortunately, we need not quote the definition to carry out the calculation of the integrals of (4.8) and in the right hand sides of (1) and (2), because the similar integrals have already calculated in [Hu]; so it is enough to quote only results in [Hu].

Now we shall calculate the integral of (4.8): the ones in the right hand sides of (1) and (2) are obtained by taking the square of it and multiplying the values replacing $\alpha$ with 0 and $2 \alpha$ respectively.

Type I. The calculation in $[\mathrm{Hu}]$, p.100-p.109 deduces that

$$
\begin{aligned}
& \int_{\Omega}\left|\psi_{f}^{(i)}(\zeta)\right|^{2}\left|\psi_{A}(x)\right|^{2+\alpha} B(\zeta, \bar{\zeta}) d \zeta
\end{aligned}
$$

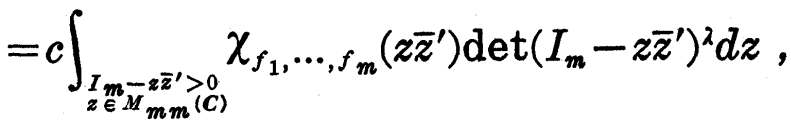

where $\lambda=(2+\alpha) l / 2-(n+m)$ and $\chi_{f_{1}, \cdots, f_{m}}(x)(x \in G L(m))$ is the trace of a representation of $G L(m)$ with signature $\left(f_{1}, \cdots, f_{m}\right)$ (see [Hu], 1.4). Then

$$
\begin{aligned}
& =c \int_{\substack{I_{m \in-x^{\prime} \bar{x}^{\prime}>0}>0 \\
x \in M_{m m}(C)}} \chi_{f_{1}+(n-m), \cdots, f_{m}+(n-m)}\left(x \bar{x}^{\prime}\right) \operatorname{det}\left(I_{m}-x \bar{x}^{\prime}\right)^{2} d x \\
& =c \operatorname{det}\left|B\left(f_{i}-i-k+(n+m)+1, \lambda+1\right)\right|_{1}^{m},
\end{aligned}
$$

where $B(\cdot, \cdot)$ is the Bessel function, $\left|a_{i k}\right|_{1}{ }^{m}$ the $(m, m)$ matrix whose $(i, k)$ 
entry equals $a_{i k}(1 \leqq i, k \leqq m)$ and $c$ does not depend on $f$ and $i$. Type II. The calculation in [Hu], p.130-p.138 deduces that

$$
\begin{aligned}
& \int_{Q}\left|\psi_{f}{ }^{(i)}(\zeta)\right|^{2}\left|\psi_{A}(x)\right|^{2+\alpha} B(\zeta, \bar{\zeta}) d \zeta \\
&= c \delta_{i_{1}}^{1} \cdots \delta_{i_{n}}^{n} B\left(f_{i_{1}}-n-i_{1}+1, \lambda+1\right) B\left(f_{i_{1}}+f_{i_{2}}-2 n-\left(i_{1}+i_{2}\right)+2, \lambda+1\right) \\
& \times \cdots \times B\left(f_{i_{1}}+\cdots+f_{i_{n}}-n^{2}-\left(i_{1}+\cdots+i_{n}\right)+n, \lambda+1\right),
\end{aligned}
$$

where $\lambda=(2+\alpha) l / 2-(n+1)$ and $c$ does not depend on $f$ and $i$.

Type III. This case is similar to Type I. We shall omit it.

Type IV. Let $z=r \xi\left(z \in C^{n}\right.$ and $\left.r=|z|\right)$. Then by [Hu], 7.4 we easily see that

$$
\begin{gathered}
\int_{\Omega}\left|\zeta \zeta^{\prime}\right|^{2 l}\left|\psi_{f-2 l}^{(i)}(\zeta)\right|^{2}\left|\psi_{A}(x)\right|^{2+\alpha} B(\zeta, \bar{\zeta}) d \zeta \\
=c B(f+n, \lambda+1),
\end{gathered}
$$

where $\lambda=(2+\alpha) l / 2-n$ and $c$ does not depend on $f$ and $i$.

In each type the value of the integral does not depend on " $i$ " ( $j$ in our notation), so the constant $C(\alpha ; 1,1 ; i, j)$ defined by the integral is independent of $j$. Moreover, since

$$
\Gamma(a) / \Gamma(a+\mu) \rightarrow a^{-\mu} \quad\left(a, \mu \in R^{+} \text {and } a \rightarrow \infty\right),
$$

the values of the integrals in the right hand sides of (1) and (2) have the same behaviour when " $f$ " ( $i$ in our notation) goes to infinity. Therefore, we can obtain the desired results.

Q.E.D.

\section{§5. Characterization of $F_{\Lambda}\left(L^{p}(G)\right)$.}

By Theorem 4.1 our problem is reduced to the case of $F_{A}\left(L^{p}(G)\right)$. As mentioned in 1.2, the answer has two forms, and the choice of the forms depends on whether $(\Lambda, p)$ is regular or not. Especially, to characterize the non regular case we need a generalization of the fractional derivatives of holomorphic functions on $\Omega$. So, first we shall define the fractional derivative $F^{[\alpha]}(\alpha \geqq 0)$ of a holomorphic function $F$ on $\Omega$ and then define a space $H_{\Lambda, \alpha}(\Omega)$ that will give a characterization of the non regular case. When $\Omega=D$ and $p=\alpha=1$, this space corresponds to $H_{1,0}(D)$ in (1.7) (see (5.2) and Remark $5.6(1)$ ).

5.1. Let $F=\sum_{l, m} a_{l m} \psi_{A m}^{l}$ be a holomorphic function on $\Omega$. Then the fractional derivative $F^{[\alpha]}$ of $F$ of order $\alpha\left(\alpha>\alpha_{1}-1\right.$ and $\left.\alpha \geqq 0\right)$ is defined formally by 


$$
F^{[\alpha]}=\sum_{l, m} c_{\alpha}^{l} \alpha_{l m} \psi_{\Delta m}^{l}
$$

where $c_{\alpha}^{l}(l \in N)$ is the constant defined by (4.8). Obviously, this definition is applicable to the case of $\alpha>\alpha_{1}-1$ and $\alpha<0$, and such an $F^{[\alpha]}$ corresponds to the fractional integral of $F$.

As an example of the fractional derivative on $\Omega$, we shall calculate $\left(T_{\Lambda}\left(g^{-1}\right) 1\right)^{[\alpha]}(z)(z \in \Omega)$ explicitly for a fixed $g$ in $G$ (see (3.4)), and then prove that it belongs to $A_{W_{A, 1, \alpha}}^{1}(\Omega)$ (see (2.9) and (2.11)) and its norm is uniformly bounded on $g \in G$. This fact will be used in the proof of the main theorem.

LEMMA 5.1. Let $\Lambda=\Lambda_{l}$ satisfy the $L^{2}$ condition and let $\alpha>\alpha_{\Lambda}-1$. Then for a fixed $g$ in $G$

$$
\left(T_{\Lambda}\left(g^{-1}\right) 1\right)^{[\alpha]}(z)=c_{\Lambda}^{2} \bar{\psi}_{\Lambda}(g) B(z, \bar{\zeta})^{(2+\alpha) l / 2 \gamma},
$$

where $\zeta=0 \cdot g$ and $z \in \Omega$.

Proof. First we shall prove that for a fixed $x \in G$

$$
S_{x}(g)=c_{\Lambda}^{-2}\left|\psi_{\Lambda}(g)\right|^{\alpha} \sum_{l, m} c_{\alpha}^{l} U_{m}^{l}(x) \bar{U}_{m}^{l}(g) \quad(g \in G)
$$

is an $L^{2}$ function on $G$ and it satisfies

$$
\bar{\psi}_{A} * S_{x}(g)=\psi_{A}\left(x g^{-1}\right)
$$

Let $S_{x}^{n}(n \in N)$ denote the partial sum of $S_{x}$ requiring that $l \leqq n$. Then by the same argument as in the proof of Lemma 4.2 it follows from (4.8) and (4.9) that

$$
\begin{aligned}
\left\|S_{x}^{n}\right\|_{2}^{2} & =c_{A}^{-4} \sum_{l \leq n}\left(c_{\alpha}^{l}\right)^{2}\left|U_{m}^{l}(x)\right|^{2} \int_{G}\left|U_{m}^{l}(g)\right|^{2}\left|\psi_{A}(g)\right|^{2 \alpha} d g \\
& =c_{A}^{-2} \sum_{l \leq n}\left(c_{\alpha}^{l}\right)^{2}\left(c_{2 \alpha}^{l}\right)^{-1}\left|U_{m}^{l}(x)\right|^{2} \\
& \sim c_{A}^{-2} \sum_{l \leq n}\left|U_{m}^{l}(x)\right|^{2} .
\end{aligned}
$$

Therefore, (2.14) implies that $S_{x}$ is an $L^{2}$ function on $G$. By the same way it follows from Lemma 2.4 (1) and (4.8) that

$$
\begin{aligned}
\bar{\Psi}_{A} * S_{x}^{n}(g) & =\int_{G} \bar{\psi}_{A}\left(g y^{-1}\right) S_{x}^{n}(y) d y \\
& =c_{A}^{-2} \sum_{l \leq n} c_{\alpha}^{l} \int_{G}\left|U_{m}^{l}(y)\right|^{2}\left|\psi_{A}(y)\right|^{\alpha} d y U_{m}^{l}(x) \bar{U}_{m}^{l}(g) \\
& =\sum_{l \leq n} U_{m}^{l}(x) \bar{U}_{m}^{l}(g) .
\end{aligned}
$$


So, Lemma $2.4(1)$ implies that $\bar{\psi}_{A} * S_{x}=\psi_{A}\left(x g^{-1}\right)$.

For all $f$ in $A_{A}^{2}(G)$ we see that

$$
\begin{aligned}
\int_{G} f(g) S_{x}(g) d g & =\int_{G} c_{A}^{-2} \psi_{A} * f(g) S_{x}(g) d g \text { by Lemma 2.4 (3) } \\
& =\int_{G} f(g) c_{A}^{-2} \bar{\psi}_{A} * S_{x}(g) d g \\
& =c_{A}^{-2} \int_{G} f(g) \psi_{A}\left(x g^{-1}\right) d g \\
& =f(g) .
\end{aligned}
$$

On the other hand, it follows from Lemma 2.4 (1) and (3.4) that

$$
T_{A}\left(g^{-1}\right) 1(z)=\psi_{A}(x)^{-1} \psi_{A}\left(x g^{-1}\right)=\psi_{A}(x)^{-1} \sum_{l, m} U_{m}^{l}(x) \bar{U}_{m}^{l}(g)
$$

for $z=0 \cdot x(x \in G)$ and thus, from Lemma $2.3(4)$, (3.5) and (5.1) that

$$
\left(T_{\Lambda}\left(g^{-1}\right) 1\right)^{[\alpha]}(z)=\psi_{\Lambda}(x)^{-1} \sum_{l, m} c_{\alpha}^{l} U_{m}^{l}(x) \bar{U}_{m}^{l}(g) .
$$

Then the above equation means that for all $F$ in $A_{A}^{2}(\Omega)$

$$
F(z)=\int_{G} F(\zeta)\left[c_{A}^{-2}\left(T_{\Lambda}\left(g^{-1}\right) 1\right)^{[\alpha]}(z) \bar{\psi}_{\Lambda}(g)^{-1}\right] B(\zeta, \bar{\zeta})^{-(2+\alpha) l / 2 \gamma+1} d \zeta .
$$

Then noting the form of $[\cdots]$ and comparing the reproducing formula in [CR], Lemma 2.1, we can obtain the desired relation. Q.E.D.

REMARK 5.2. Lemma 3.6 is nothing but the case of $\alpha=0$ in Lemma 5.1.

Proposition 5.3. Let $\Lambda=\Lambda_{l}$ satisfy the $L^{2}$ condition and $\alpha>\alpha_{\Lambda}$. Then

$$
\sup _{g \in G}\left\|\left(T_{\Lambda}\left(g^{-1}\right) 1\right)^{[\alpha]}\right\|_{1, W_{A, 1, \alpha}}<\infty,
$$

(see (2.9) and (2.11)).

Proof. By Lemmas 5.1 and 3.1 it is enough to prove that

$$
\sup _{\zeta \in \Omega} B(\zeta, \bar{\zeta})^{-\beta} \int_{\Omega}|B(z, \bar{\zeta})|^{(2+\alpha) \beta} B(z, \bar{z})^{-(1+\alpha) \beta+1} d z<\infty,
$$

where $\beta=l / 2 \gamma$. Then this is clear from Lemma 3.4.

Q.E.D.

5.2. We shall define $H_{\lambda, \alpha}^{p}(\Omega)\left(1 \leqq p \leqq 2\right.$ and $\left.\alpha>\alpha_{A}-1\right)$ as follows: 


$$
\begin{aligned}
H_{A, \alpha}^{p}(\Omega)=\{F: \Omega \rightarrow C & \text { (1) } F \text { is holomorphic on } \Omega, \\
& \text { (2) } \left.F^{[\alpha]} \text { belongs to } A_{W_{A, p, \alpha}^{p}}^{p}(\Omega)\right\} .
\end{aligned}
$$

Obviously, since $F^{[0]}=F$,

$$
A_{\Lambda}^{p}(\Omega)=A_{\Lambda, 0}^{p}(\Omega)
$$

(see (2.12)). Especially, when $p=2$, we see the following

Proposition 5.4. Let $\Lambda=\Lambda_{l}$ satisfy the $L^{2}$ condition and $\alpha>\alpha_{\Lambda}-1$. Then $A_{\Lambda}^{2}(\Omega)=H_{\Lambda, \alpha}^{2}(\Omega)$, actually for all $F$ in $A_{\Lambda}^{2}(\Omega)$

$$
\|F\|_{2, W_{\Lambda, 2,0}} \sim\left\|F^{[\alpha]}\right\|_{2, W_{\Lambda, 2, \alpha}},
$$

where " " means that the ratio of the left and the right is bounded above and below by positive constants which do not depend on $F$.

Proof. Let $F=\sum_{l, m} a_{l m} \psi_{m}^{l}$ be in $A_{\Lambda}^{2}(\Omega)$. Then

$$
\begin{aligned}
\left\|F^{[\alpha]}\right\|_{2, W_{A, 2, \alpha}} & =\int_{\Omega}\left|\sum_{l, m} c_{\alpha}^{l} a_{l m} \psi_{m}^{l}(\zeta)\right|^{2}\left|\psi_{\Lambda}(x)\right|^{2(1+\alpha)} B(\zeta, \bar{\zeta}) d \zeta,
\end{aligned}
$$

where $\zeta=0 \cdot x$. Then rewriting this integral over $\Omega$ as the one over $G$ (see (2.10)) and applying the same argument as in the proof of Lemma 4.2 , we can deduce that

$$
\begin{aligned}
& =\sum_{l, m}\left(c_{\alpha}^{l}\right)^{2}\left|a_{l m}\right|^{2} \int_{\alpha}\left|\psi_{m}^{l}(\zeta)\right|^{2}\left|\psi_{\Lambda}(x)\right|^{2(1+\alpha)} d \dot{g} \\
& =c_{A}{ }^{2} \sum_{l, m}\left(c_{\alpha}^{l}\right)^{2}\left|a_{l m}\right|^{2}\left(c_{2 \alpha}^{l}\right)^{-1} \quad \text { by (3.3), (3.5), (4.8) } \\
& \sim \sum_{l, m}\left|a_{l m}\right|^{2} \quad \text { by (4.9) } \\
& =\left\|F^{\prime}\right\|_{2, W_{\Lambda, 2,0}} \text {. }
\end{aligned}
$$

Now we shall state our main theorem.

THEOREM 5.5. Let $A=\Lambda_{l}$ satisfy the $L^{2}$ condition and $1 \leqq p \leqq 2$. Then

(1) If $(\Lambda, p)$ is regular, then $F_{\Lambda}\left(L^{p}(G)\right)=A_{\Lambda}^{p}(\Omega)$ and $F_{\Lambda}: P_{\Lambda}\left(L^{p}(G)\right) \rightarrow$ $A_{\Lambda}^{p}(\Omega)$ is bijective and norm preserving.

(2) If $(\Lambda, p)$ is not regular, then $F_{\Lambda}\left(L^{p}(G)\right)=H_{\Lambda, \alpha}^{p}(\Omega)$ for all $\alpha>\alpha_{\Lambda}$ and $F_{\Lambda}: P_{\Lambda}\left(L^{p}(G)\right) \rightarrow H_{\Lambda, \alpha}^{p}(\Omega)$ is bijective.

Proof. (1) We note that $F_{A}\left(L^{p}(G)\right)=F_{A}\left(P_{A}\left(L^{p}(G)\right)\right)$ by (4.6) and, since $(\Lambda, p)$ is regular, $F_{\Lambda}=I_{A}$ on $P_{\Lambda}\left(L^{p}(G)\right)=A_{A}^{p}(G)$ by (4.2) and (4.7). Therefore, (1) is nothing but Proposition 3.9. 
(2) It follows from Table 4 that if $(\Lambda, p)$ is non regular $(\Lambda, 1)$ is also non regular. So, we shall prove this case first.

The case of $(\Lambda, 1)$ : Let $f$ be in $L^{1}(G)$. Then by Proposition 5.3 we see that for $\alpha>\alpha_{A}$

$$
\begin{aligned}
\left\|\left(F_{A}(f)\right)^{[\alpha]}\right\|_{1, W_{A, 1, \alpha}} & \leqq c_{A}^{-1} \int_{G}|f(g)|\left\|\left(T_{A}\left(g^{-1}\right) 1\right)^{[\alpha]}\right\|_{1, W_{A, 1} \alpha} d g \\
& \leqq c\|f\|_{1}<\infty .
\end{aligned}
$$

This means that $F_{\Lambda}(f) \in H_{\Lambda, \alpha}^{1}(\Omega)$ and thus, $F_{A}\left(L^{1}(G)\right)$ is contained in $H_{\lambda, \alpha}^{1}(\Omega)$.

Conversely, let $F=\sum_{l, m} a_{l m} \psi_{m}^{l}$ be in $H_{\Lambda, \alpha}^{1}(\Omega)\left(\alpha>\alpha_{A}\right)$, and let

$$
f(g)=\sum_{l, m} a_{l m} U_{m}^{l}(g)=c_{A}^{-1} \psi_{\Lambda}(g) F(z)
$$

and

$$
\begin{aligned}
{[f](g) } & =\sum_{l, m} a_{l m}\left[U_{m}^{l}\right](g) \\
& =\left|\psi_{\Lambda}(g)\right|^{\alpha} \sum_{l, m} c_{m}^{l} a_{l m} U_{m}^{l}(g) \\
& =c_{\Lambda}^{-1}\left|\psi_{\Lambda}(g)\right|^{\alpha} \psi_{\Lambda}(g) F^{[\alpha]}(z),
\end{aligned}
$$

where $z=0 \cdot g$ (see Lemmas 4.2 and 4.4). Then since $F^{[\alpha]}$ belongs to $A_{W_{A, 1, \alpha}}^{1}(\Omega)$, it is easy to see that

$$
\|[f]\|_{1}=c_{A}\left\|F^{[\alpha]}\right\|_{1, w_{A, 1, \alpha}}<\infty .
$$

Since $\alpha>\alpha_{1}$, it follows from (2.11) and (3.2) that $A_{W_{A, 1, \alpha}}^{2}(\Omega)$ is contained in $A_{W_{1,1}, \alpha}^{1}(\Omega)$ and moreover, it is a dense subspace, for $A_{W_{1,1, \alpha}}^{2}(\Omega)$ contains all functions holomorphic in a domain bigger than $\Omega$ and such functions are dense in $A_{W_{A, 1}, \alpha}^{1}(\Omega)$. Therefore there exists a sequence $\left\{F_{n}\right\}$ of holomorphic functions on $\Omega$ such that $F_{n}^{[\alpha]} \in A_{W_{A, 1}, \alpha}^{2}(\Omega)$ and $\left\|F^{[\alpha]}-F_{n}^{[\alpha]}\right\|_{1, w_{A, 1, \alpha}} \rightarrow 0(n \rightarrow \infty)$. Especially, we can deduce that $F_{n}^{[\alpha]}$ converges to $F^{[\alpha]}$ pointwisely on $\Omega$ (cf. [DRS], Theorem 3 (i)). Now let $f_{n}$ and $\left[f_{n}\right]$ denote the functions on $G$ which are respectively given by replacing $F$ in (5.4) with $F_{n}$. Then as above we see that $\left\|[f]-\left[f_{n}\right]\right\|_{1} \rightarrow 0$ $(n \rightarrow \infty)$ and $\left[f_{n}\right]$ converges to $[f]$ pointwisely on $G$.

Now we recall that $\left|\psi_{1}\right| \leqq 1$ (see Lemma 3.1), and thus each $F_{n}^{[\alpha]}$ belongs to $A_{W_{A, 2, \alpha}}^{2}(\Omega)$. Then by Proposition 5.4 we see that $F_{n} \in A_{\Lambda}^{2}(\Omega)$ and $f_{n} \in L^{2}(G)$ (see (5.4)). Therefore, applying the same argument used in the proofs of Lemmas 4.2 and 5.1, we can deduce that

$$
c_{A}^{-1} \psi_{\Lambda} *\left[f_{n}\right]=c_{A} f_{n} \text {. }
$$


Since $\left\|\psi_{\Lambda} *\left([f]-\left[f_{n}\right]\right)\right\|^{2} \leqq\left\|\psi_{1}\right\|_{2}\left\|[f]-\left[f_{n}\right]\right\|_{1} \rightarrow 0$ when $n \rightarrow \infty, c_{A} f_{n}$ converges to $c_{A}{ }^{-1} \psi_{A} *[f]$, say $h$ for simplicity, in $L^{2}$ norm. Then $c_{A} F_{n}{ }^{[\alpha]}$ converges to $F_{\Lambda}(h)^{[\alpha]}$ in $H_{\lambda, \alpha}^{2}(\Omega)$ by Proposition 5.4, and thus it converges pointwisely (cf. [DRS], Theorem $3(\mathrm{i})$ ), so we can obtain that $c_{A} F^{[\alpha]}=F_{A}(h)^{[\alpha]}$. Since $c_{A}^{-1} h \in L^{2}(G)$ is a discrete part of $[f]$, the first case $(1)(p=2)$ means that it is of the form $\sum_{l, m} b_{l m} U_{m}^{l}(g)$. Then $h$ must be equal to $c_{1} f$ and it follows that

$$
c_{\Lambda}^{-1} \psi_{A} *[f]=c_{A} f
$$

Especially,

$$
F_{A}([f])=I_{A}\left(\phi_{1}^{1} *[f]\right)=c_{A} I_{A}(f)=F .
$$

This means that $F \in F_{A}\left(L^{1}(G)\right)$ and thus, $H_{A, \alpha}^{1}(\Omega)$ is contained in $F_{A}\left(L^{1}(G)\right.$ ). Therefore, we conclude that $F_{A}\left(L^{1}(G)\right)=H_{A, \alpha}^{1}(\Omega)$ for all $\alpha>\alpha_{A}$, and $F_{A}$ is bijective on $P_{A}\left(L^{1}(G)\right)$ by (4.4).

This completes the proof of the case of $(\Lambda, 1)$.

The general case of non regular $(\Lambda, p)$ : By the same way as in the previous case of $(\Lambda, 1)$ we see that $H_{\Lambda, \alpha}^{p}(\Omega)$ is contained in $F_{\Lambda}\left(L^{p}(G)\right)$ for all $\alpha>\alpha_{A}$, where in the argument of the $L^{2}$ convergence of $c_{A}^{-1} \psi_{A} *\left[f_{n}\right]$ we use the Kunze-Stein phenomenon (see [C]). Therefore, by noting (4.4) in order to accomplish the proof of the theorem it is enough to show that $F_{A}\left(L^{p}(G)\right) \subset H_{\Lambda, \alpha}^{p}(\Omega)$. Actually, this fact will be shown by an interpolation argument as follows.

Since $\Lambda$ satisfies the $L^{2}$ condition, but not the one of $L^{1}, \alpha_{1}-1$ is negative (see Remark 3.2), so it follows from Lemma 4.4 that the fractional derivative of order $\alpha$ (see (5.1)) is defined for $\alpha \geqq 0$. Then for an $L^{p}(1 \leqq q \leqq 2)$ function $f$ on $G$ we can define the operator $K_{\alpha}(\alpha \geqq 0)$ as follows:

$$
K_{\alpha}: f \longmapsto\left(F_{\Lambda}(f)\right)^{[\alpha]} .
$$

As shown in the case of $(\Lambda, 1)$, if $\alpha>\alpha_{\Lambda}, K_{\alpha}$ is bounded of $L^{1}(G)$ into $A_{W_{A, 1, \alpha}}^{1}(\Omega)$. On the other hand, since $(\Lambda, 2)$ is regular, (1) implies that $K_{0}$ is bounded of $L^{2}(G)$ into $A_{\Lambda}^{2}(\Omega)=A_{W_{\Lambda, 2}, 0}^{2}(\Omega)$. Then by Proposition 5.4 we see that

$$
\left\|K_{\alpha}(f)\right\|_{2, W_{A, 2, \alpha}}=\left\|K_{0}(f)\right\|_{2, W_{A, 2,0}} \leqq c\|f\|_{2} .
$$

This means that $K_{\alpha}\left(\alpha>\alpha_{A}\right)$ is also bounded of $L^{2}(G)$ into $A_{W_{A, 2, \alpha}}^{2}(\Omega)$. Therefore, the interpolation argument (cf. [BL], p. 17) deduces that $K_{\alpha}$ is bounded of $L^{p}(G)$ into $A_{W_{\Lambda, p, \alpha}^{p}}^{p}(\Omega)(1<p<2)$, and then $F_{\Lambda}\left(L^{p}(G)\right) \subset H_{\Lambda, \alpha}^{p}(\Omega)$. 
REMARK 5.6. (1) Theorem 5.5 (2) implies that $H_{A, \alpha}^{p}(\Omega)\left(\alpha>\alpha_{A}\right)$ does not depend on $\alpha$. Therefore, we can put

$$
H_{\Lambda, *}^{p}(\Omega)=H_{\Lambda, \alpha}^{p}(\Omega) \quad\left(\alpha>\alpha_{\Lambda}\right)
$$

Moreover, we easily see that the proof in the case of non regular is also applicable to the case of regular, and thus, Theorem $5.5(2)$ is valid for all $(\Lambda, p)$. In particular,

$$
\text { if }(\Lambda, p) \text { is regular, then } A_{\Lambda}^{p}(\Omega)=H_{\Lambda, *}^{p}(\Omega) \text {. }
$$

(2) As said in $\S 4$ the discrete part of $L^{p}(G)(1 \leqq p \leqq 2)$ is contained in $L^{2}(G)$. Therefore, it follows from Theorem 5.5 that

$$
H_{\Lambda, *}^{p}(\Omega) \subset A_{\Lambda}^{2}(\Omega) \text {. }
$$

(3) Let $(\Lambda, p)\left(\Lambda=\Lambda_{l}, l \in Z\right)$ be regular and $F$ be in $A_{\Lambda}^{p}(\Omega)$. Then Theorem 5.5 (1) means the reproducing formula for $F$, that is,

$$
F(z)=\int_{\Omega} F(\zeta) B(z, \bar{\zeta})^{l / r} B(\zeta, \bar{\zeta})^{-l / \tau+1} d \zeta
$$

(see Lemma $2.4(3)$, (3.3) and (4.2)). Let $F$ be in $H_{\Lambda, *}^{1}(\Omega)$ and let $f$ denote an $L^{1}$ function on $G$ such that $F_{A}(f)=F$. Then noting the proof of the case $(\Lambda, 1)$ in Theorem 5.5 , we can deduce that for $\alpha>\alpha_{\Lambda}$

$$
\begin{aligned}
F^{[\alpha]}(z) & =c_{A}^{-2} \int_{G} f(g)\left(T_{A}\left(g^{-1}\right) 1\right)^{[\alpha]}(z) d g \\
& =\int_{0} F(\zeta) B(z, \bar{\zeta})^{(2+\alpha) t / 2 \gamma} B(\zeta, \bar{\zeta})^{-l / \gamma+1} d \zeta
\end{aligned}
$$

(see Lemmas 3.1 and 5.1) and then $F^{[\alpha]} \in A_{W_{A, 1, \alpha}}^{1}(\Omega)$ by Theorem $5.5(2)$.

(4) Let us suppose that $F$ is in $A_{\Lambda}^{p}(\Omega)=A_{W_{A, p}, 0}^{p}(\Omega)(1<p<2)$ and $l>2(\gamma-1) / p$. Then applying Lemma 3.5, in which we take $r$ and $\theta$ as $(1+\alpha) l p / 2 \gamma-1$ and $-\alpha l p / 2 \gamma$ respectively, we see that the integral formula (5.6) is valid for $2(\gamma-1) / l p-1<\alpha<(2-p) /(p-1)$ and $F^{[\alpha]}$ is contained in $A_{W_{\Lambda}, p, \alpha}^{p}(\Omega)$. Here we note $2(\gamma-1) / l p-1<0<\alpha_{\Lambda^{\bullet}}$ Roughly speaking, this result corresponds to an interpolation between (5.5) and (5.6); however, for $F$ in $A_{A}^{2}(\Omega)(p=2)$ the fractional derivative $F^{[\alpha]}$ of $F$ of order $\alpha>0$ could not be defined by the integral formula (5.6). Therefore, in $\S 5$ we adopted the definition (5.1) without using the integral formula.

(5) In the proof of Theorem 5.5 (2) we used the interpolation argument between $(\Lambda, 1)$ and $(\Lambda, 2)$, so we need the condition $\alpha>\alpha_{\Lambda}$ that is necessary for the boundedness of $K_{\alpha}$ in the case of $(\Lambda, 1)$. Therefore, 
if we could prove the assertion in Theorem 5.5(2) without using the interpolation argument, we may weaken the condition of $\alpha$.

CONJECTURE. In the statement of Theorem $5.5(2)$ we can replace the condition $\alpha>\alpha_{A}$ by $\alpha>\left(\alpha_{A}+1\right) / p-1$.

\section{§6. Application.}

We shall obtain some properties of the fractional derivatives of holomorphic functions on $\Omega$. In the previous sections the parameter $l$ must be integer, for $\Lambda_{l}$ is an integral form on $\mathfrak{h}_{c}$.

6.1. We shall consider the continuation of the parameter. Let $l, \alpha$ and $p \in R$ and we put

$$
w_{l, p, \alpha}(z)=B(z, \bar{z})^{-(1+\alpha) l_{p} / 2 \gamma+1}
$$

(cf. (2.11) and Lemma 3.1). Then as noted in Remark $5.6(3)$ and (4), for $F$ in $A_{W l, p, 0}^{p}(\Omega)$ the fractional derivative $F^{[\alpha]}$ of $F$ can be defined by the integral formula (5.6) for which the integral exists. Especially, $l$ needs not be integral, and when $p=1$, Lemma 3.4 implies that $F^{[\alpha]}$ is well-defined for all $\alpha>2(\gamma-1) / l-1$ and it is contained in $A_{W_{l, 1, \alpha}}^{1}(\Omega)$. Therefore, combining with Theorem 5.5(1), we see that

THEOREM 6.1. Let $l \in R, 1 \leqq p \leqq 2$ and let $F$ be in $A_{w_{l, p, \alpha}}^{p}(\Omega)$.

(1) If $(\Lambda, p)(l \in Z)$ is regular or $p=1$ and $l>2(\gamma-1)$ then $F^{[\alpha]}$ belongs to $A_{W_{l, p}, \alpha}^{p}(\Omega)$ for all $\alpha \geqq 0$.

(2) If $l>2(\gamma-1) / p$ and $1<p<2$, then $F^{[\alpha]}$ belongs to $A_{W_{l, p}, \alpha}^{p}(\Omega)$ for all $0 \leqq \alpha<(2-p) /(p-1)$.

6.2. We shall consider the case of $G=S U(1,1)$ and $\Omega=D^{n}$. Let

$$
F(z)=\sum_{i, j} a_{i j} \psi_{j}^{i}(z) \quad\left(z \in D^{n}\right)
$$

be a holomorphic function on $D^{n}$. Then referring to the calculation of Type I in Lemma 4.4, we see that the fractional derivative of $F^{[\alpha]}$ of $F$ of order $\alpha$, where $\alpha>\alpha_{1}=2 n / l-1$, is given by

$$
F^{[\alpha]}(z)=C_{n, l, \alpha} \sum_{i, j} \Gamma(i+l+(1 / 2) \alpha l) \Gamma(i+l)^{-1} a_{i j} \psi_{j}^{\beta}(z),
$$

where the constant $C_{n, l, \alpha}$ does not depend on $i$ and $j$. Here we put

$$
F^{(\beta)}(z)=F^{[2 \beta / l]}(z) \quad(\beta \geqq 0) .
$$


Then, when $n=1$ and $\Omega=D$, if we take $l>2$ and $p=1$ in Theorem 6.1 (1), we can deduce Theorem 5 in [DRS]:

Proposition 6.2 ([DRS]). If $F$ belongs to $A_{W_{l, 1,0}}^{1}(D)(l>2)$, then $F^{(\beta)}$ belongs to $A_{W_{l, 1,2 \beta}}^{2}(D)$.

If we take $\alpha=2 / l$ in Theorem 6.1, we see that

Proposition 6.3. Let $1<p<2$ and $p>2 n / l$. Suppose that $l \in N$ or $p<2(l+1) /(l+2)$. Then if a holomorphic function $F$ on $D^{n}$ satisfies

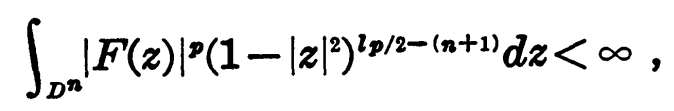

the derivative $F^{(1)}$ satisfies

$$
\int_{D^{n}}\left|F^{(1)}(z)\right|^{p}\left(1-|z|^{2}\right)^{(l+2) / 2 / 2-(n+1)} d z<\infty
$$

Add in the proof.

(1) For a holomorphic function $F$ on $\Omega$ the fractional derivative $F^{[\alpha]}$ of $F$ is defined by (5.1) in which we use the constants given by (4.8). One of the reason we adopt this definition is, when $\Omega=D$, a modification $F^{(\alpha)}$ of $F^{[\alpha]}$ (see (6.3)) coincides with the classical fractional derivative defined in [DRS], p. 35. On the other hand, if we replace " $\left|\psi_{A}(x)\right|^{\alpha}$ in (4.8) by a positive spherical function $\Psi(x)$ on $G$ satisfying

$$
\Psi(x) \psi_{A}(x)^{2} \in L^{1}(G)
$$

(cf. (3.2)), we can define a new fractional derivative by using the constants cl given by

$$
c_{\Psi}^{i} \int_{G}\left|U_{j}^{i}(x)\right|^{2} \Psi(x) d x=c_{A}^{2} .
$$

Clearly, if Lemma 4.4 is valid for $c^{\ddagger}$, the whole results in $\$ 5$ and $\S 6$ are also valid for this type of the fractional derivative.

(2) In $\S 3$ and the succeeding sections we assume that $\Omega$ is one of the classical bounded symmetric domains listed in Table 1 . We need this assumption essentially in the calculation in Lemma 4.4 adopted from [Hu]. Other results related with Bergman kernel can be generalized to arbitrary bounded symmetric domains (cf. [RV] and [VR]).

ACKNOWLEDGEMENT. The author would like to thank the referee for suggestions on improving the exposition of the paper and valuable comments. 


\section{References}

[BL] J. BERGH and J. LÖFSTRÖM, Interpolation Spaces, Springer-Verlag, 1976.

[C] M. Cowling, The Kunze-Stein phenomenon, Ann. of Math., 107 (1978), 209-234.

[CR] R. R. CoIFMAN and R. RochBeRg, Representation theorems for holomorphic and harmonic functions in $L^{p}$, Asterisque, 77 (1980), $12-66$.

[CS] J.R. CLERC and E.M. STEIN, $L^{p}$-multipliers for non-compact symmetric spaces, Proc. Nat. Acad. Sci. U.S.A., 71 (1974), 3911-3912.

[DRS] P. L. DUREN, B. W. Romberg and A. L. SHIELDS, Linear functionals on $H^{p}$ spaces with $0<p<1$, J. Reine Angew. Math., 238 (1969), 32-60.

[HC] HARISH-ChandRA, Representations of semisimple Lie groups VI, Amer. J. Math., 78 (1956), 564-628.

[HC2] HARISH-ChANDRA, Harmonic analysis on real reductive groups III. The Maass-Selberg relations and the Plancherel formula, Ann. of Math., 104 (1976), 117-201.

[He] S. Helgason, Groups and Geometric Analysis, Academic Press, 1984.

[Hu] L. K. HUA, Harmonic Analysis of Functions of Several Complex Variables in the Classical Domains, Amer. Math. Soc., 1963.

[Ka] T. KawazoE, Fourier coefficients of $L^{p}$ functions on $S U(1,1)$, Keio Univ. Res. Rep., 7 (1985); Discrete part of $L^{p}$ functions on $S U(1,1)$, Keio Univ. Res. Rep., 10 (1986).

[Kn] A. W. KNAPP, Bounded symmetric domains and holomorphic discrete series, Pure and Applied Math., 8 (1972), 211-246, Marcel Dekker.

[KS] R. A. KUNZE and E. M. STEIN, Uniformly bounded representations and harmonic analysis of the $2 \times 2$ real unimodular group, Amer. J. Math., 82 (1960), 1-62.

[M] Y. MUTA, On spherical functions with one dimensional $K$-types and the Paley-Wiener type theorem on some simple Lie groups, Rep. Fac. Sci. Eng. Saga Univ., 9 (1981), 31-59.

[RV] H. RossI and M. VERGNE, Representations of certain solvable Lie groups on Hilbert spaces of holomorphic functions and applications to holomorphic discrete series of a semi-simple Lie group, J. Funct. Anal., 13 (1973), 324-389.

[Su] M. SUGIURA, Unitary Representations and Harmonic Analysis, Wiley, New York, 1975.

[Va] V.S. Varadarajan, Harmonic Analysis on Reductive Groups, Lecture Notes in Math., 576 (1977), Springer.

[VR] M. VERGNe and H. Rossi, Analytic continuation of the holomorphic discrete series of a semisimple Lie group, Acta Math., 136 (1976), 1-59.

[Wa] G. WARNER, Harmonic Analysis on Semi-Simple Lie Groups II, Springer, 1972.

Present Address:

Department of Mathematics, Faculty of Science and Technology, Keio University HIYOSHI, KoHOKU-KU, YokOHAMA 223, JAPAN 